\title{
The ATLASGAL survey: a catalog of dust condensations in the Galactic plane ${ }^{\star}$
}

\author{
T. Csengeri ${ }^{1}$, J. S. Urquhart ${ }^{1}$, F. Schuller ${ }^{2}$, F. Motte $^{3}$, S. Bontemps ${ }^{4}$, F. Wyrowski ${ }^{1}$, K. M. Menten ${ }^{1}$, L. Bronfman ${ }^{5}$ \\ H. Beuther ${ }^{6}$, Th. Henning ${ }^{6}$, L. Testi ${ }^{7,8}$, A. Zavagno ${ }^{9}$, and M. Walmsley ${ }^{8,10}$ \\ 1 Max Planck Institute for Radioastronomy, Auf dem Hügel 69, 53121 Bonn, Germany \\ e-mail: ctimea@mpifr-bonn.mpg.de \\ 2 European Southern Observatory, 3107 Alonso de Cordova, Vitacura, Santiago, Chile \\ ${ }^{3}$ Laboratoire AIM Paris Saclay, CEA-INSU/CNRS-Université Paris Diderot, IRFU/SAp CEA-Saclay, 91191 Gif-sur-Yvette, France \\ ${ }^{4}$ OASU/LAB-UMR5804, CNRS, Université Bordeaux 1, 33270 Floirac, France \\ 5 Departamento de Astronomía, Universidad de Chile, Casilla 36-D Santiago, Chile \\ ${ }^{6}$ Max Planck Institute for Astronomy, Königstuhl 17, 69117 Heidelberg, Germany \\ 7 European Southern Observatory, Karl-Schwarzschild-Strasse 2, 85748 Garching, Germany \\ 8 INAF - Osservatorio Astrofisico di Arcetri, Largo E. Fermi 5, 50125 Firenze, Italy \\ 9 Aix Marseille Université, CNRS, LAM (Laboratoire d'Astrophysique de Marseille), UMR 7326, 13388 Marseille, France \\ 10 Dublin Institute for Advanced Studies, Burlington Road 10, Dublin 4, Ireland
}

Received 2 August 2013 / Accepted 27 February 2014

\section{ABSTRACT}

\begin{abstract}
Context. The formation processes and the evolutionary stages of high-mass stars are poorly understood compared to low-mass stars. Large-scale surveys are needed to provide an unbiased census of high column density sites that can potentially host precursors to high-mass stars.

Aims. The ATLASGAL survey covers 420 sq. degree of the Galactic plane, between $-80^{\circ}<\ell<+60^{\circ}$ at $870 \mu$ m. Here we identify the population of embedded sources throughout the inner Galaxy. With this catalog we first investigate the general statistical properties of dust condensations in terms of their observed parameters, such as flux density and angular size. Then using mid-infrared surveys we aim to investigate their star formation activity and the Galactic distribution of star-forming and quiescent clumps. Our ultimate goal is to determine the statistical properties of quiescent and star-forming clumps within the Galaxy and to constrain the star formation processes.

Methods. We optimized the source extraction method, referred to as MRE-GCL, for the ATLASGAL maps in order to generate a catalog of compact sources. This technique is based on multiscale filtering to remove extended emission from clouds to better determine the parameters corresponding to the embedded compact sources. In a second step we extracted the sources by fitting 2D Gaussians with the Gaussclumps algorithm.

Results. We have identified in total 10861 compact submillimeter sources with fluxes above $5 \sigma$. Completeness tests show that this catalog is $97 \%$ complete above $5 \sigma$ and $>99 \%$ complete above $7 \sigma$. Correlating this sample of clumps with mid-infrared point source catalogs (MSX at $21.3 \mu \mathrm{m}$ and WISE at $22 \mu \mathrm{m}$ ), we have determined a lower limit of $33 \%$ that is associated with embedded protostellar objects. We note that the proportion of clumps associated with mid-infrared sources increases with increasing flux density, achieving a rather constant fraction of $\sim 75 \%$ of all clumps with fluxes over $5 \mathrm{Jy} /$ beam being associated with star formation. Examining the source counts as a function of Galactic longitude, we are able to identify the most prominent star-forming regions in the Galaxy.

Conclusions. We present here the compact source catalog of the full ATLASGAL survey and investigate their characteristic properties. From the fraction of the likely massive quiescent clumps ( $25 \%)$, we estimate a formation time scale of $\sim 7.5 \pm 2.5 \times 10^{4} \mathrm{yr}$ for the deeply embedded phase before the emergence of luminous young stellar objects. Such a short duration for the formation of high-mass stars in massive clumps clearly proves that the earliest phases have to be dynamic with supersonic motions.
\end{abstract}

Key words. surveys - stars: formation - stars: massive - ISM: structure - Galaxy: structure

\section{Introduction}

The dominant formation mechanism leading to the birth of high-mass stars is still an enigma in modern astrophysics. Unlike for low-mass stars, there is no clear, observationally constrained evolutionary sequence for individual protostars above $10 M_{\odot}$, because they are rare and likely to evolve on short time scales, therefore it is challenging to identify and study them.

* Full Table 1 is only available at the CDS via anonymous ftp to cdsarc.u-strasbg. fr (130.79.128.5) or via

http://cdsarc.u-strasbg.fr/viz-bin/qcat?J/A+A/565/A75
Nevertheless, they are fundamental building blocks of galaxies, because they provide significant mechanical and radiative feedback to the interstellar medium and enrich it with heavy elements. They are used as a tool for studying star formation and the evolution of galaxies in various environments as a function of redshift (Kennicutt 1998), so it is crucial to study them first locally in our Galaxy in much greater detail.

Observations of high-mass stars are greatly hindered because they are still deeply embedded in their dust cocoons when reaching the main sequence. Since star formation in general proceeds in the densest regions of molecular clouds, dust is the best tracer for identifying these locations (see Evans 1999, for a review). In 
fact the first systematic surveys for massive young stellar objects (MYSOs) have used the infrared emission of heated dust to pin down the most luminous sites in our Galaxy.

These studies used the IRAS survey (Hughes \& MacLeod 1989; Wood \& Churchwell 1989; Bronfman et al. 1996; Molinari et al. 1996) to look for H II regions and MYSOs (Sridharan et al. 2002; Beuther et al. 2002) based on their infrared colors. These samples were then extended using data from the more sensitive Midcourse Space Experiment (MSX; Price et al. 2001) point source catalog (Egan et al. 2003). One notable example is the Red MSX Source (RMS; Hoare et al. 2005) Survey, which used a combination of near- and mid-infrared colors to identify a large sample of MYSO candidates (Lumsden et al. 2002). Their initial sample was, however, contaminated by asymptotic giant branch (AGB) stars and evolved stars whose mass loss has stopped and dust shells became detached (postAGB stars and planetary nebulae). These contaminating sources have been identified through a set of multiwavelength observations (e.g., Urquhart et al. 2008) and were removed from the final sample (Lumsden et al. 2013). However, since the infrared emission traces heated dust, this sample is strongly biased toward the more evolved MYSO and H II region phases of high-mass star formation.

The search for the earlier, hence colder, stages began with the discovery of infrared-dark clouds (IRDCs), which trace high dust column density (Perault et al. 1996; Egan et al. 1998; Carey et al. 1998). These clouds have been cataloged using MSX (Simon et al. 2006; Rathborne et al. 2006) and the more sensitive Spitzer Space Telescope (Peretto \& Fuller 2009, 2010; Butler $\&$ Tan 2009; Rygl et al. 2010). Their physical properties have also been extensively studied since then (e.g., Pillai et al. 2006; Vasyunina et al. 2009; Ragan et al. 2012). Although IRDCs have been considered for a long time to be the initial stages for highmass star formation, some of them have been shown to simply be holes in the interstellar medium (Wilcock et al. 2012), and in fact only a small fraction of them is likely to sustain massive star formation (Peretto et al. 2010; Kauffmann \& Pillai 2010). As a consequence, IRDCs also represent a biased and incomplete sample of high-mass star-forming sites.

For unambiguously tracing high column densities, the optically thin emission from dust in the millimeter/submillimeter regime is the best tool (André et al. 2000). Since it is sensitive to both cold and warm dust, it is the least biased tracer of all embedded evolutionary stages of star formation. Such studies were performed first on smaller areas of the sky in nearby low-mass starforming regions until a few years ago (e.g., Motte et al. 1998, 2007, 2001; Johnstone et al. 2000, 2001; Enoch et al. 2007); however, larger area surveys are required to reveal statistically significant samples of high column density sites. Thanks to the recent development of large field of view bolometer cameras, surveys covering a substantial fraction of the Galactic plane became feasible. The ultimate goal of unbiased surveys is to identify (e.g., Motte et al. 2007; Schuller et al. 2009; Molinari et al. 2010b; Aguirre et al. 2011) and then characterize large samples of high-mass star-forming sites (e.g., Dunham et al. 2011a,b; Wienen et al. 2012; Urquhart et al. 2013a). Statistical studies are required to then constrain the evolutionary stages of high-mass star formation and reveal the corresponding time scales for these stages. Furthermore, such large-scale surveys allow the properties of star-forming sites in various environments and conditions to be studied so that the dominant processes regulating highmass star formation can be pinned down. Here we present results of one of these large-scale surveys, the APEX Telescope Large Area Survey of the Galaxy (ATLASGAL).
The ATLASGAL survey ${ }^{1}$ (Schuller et al. 2009) imaged the Galactic plane between Galactic longitude, $-60^{\circ} \leq \ell \leq+60^{\circ}$ and Galactic latitude $-1.5^{\circ} \leq b \leq+1.5^{\circ}$ at $870 \mu \mathrm{m}$ with the LABOCA camera (Siringo et al. 2009) on the APEX Telescope (Güsten et al. 2006) in its first campaign. In a subsequent step, an extension towards Galactic longitude $-80^{\circ} \leq \ell \leq-60^{\circ}$ and Galactic latitude $-2^{\circ} \leq b \leq+1.0^{\circ}$ was added. All together, the total area of the survey covers $\sim 420$ sq. degree of the Galactic plane at a 19.2 spatial resolution.

The ATLASGAL survey supersedes other ground-based surveys providing the most sensitive and complete view of the inner Galaxy at submillimeter wavelengths. So far only spacebased missions provide better sensitivity. In this context the ATLASGAL survey is outstanding because it provides a view of the thermal dust emission at a comparable angular resolution at $870 \mu \mathrm{m}$ to Herschel at $250 \mu \mathrm{m}$ and a $2 \times$ better spatial resolution than Herschel at $500 \mu \mathrm{m}$, where the dust emission is optically thin. It is therefore well suited to studying deeply embedded objects, the majority of which are sites of ongoing star formation.

ATLASGAL is complemented well by other surveys of dust in the Galactic plane at various wavelengths, such as the midinfrared surveys with Spitzer (GLIMPSE at 3.6, 4.8, 5.6, and $8 \mu \mathrm{m}$ Benjamin et al. 2003 and MIPSGAL at $24 \mu \mathrm{m}$, Carey et al. 2009) and WISE (3.6, 4.6, 11.8, and $22 \mu \mathrm{m}$, Wright et al. 2010) probing the warm dust. The Hi-Gal survey (Herschel Infrared GALactic plane survey; Molinari et al. 2010b), uses the PACS and SPIRE instruments onboard Herschel to map the Galactic plane with unprecedented sensitivity from the far-infrared to the submillimeter wavelength regime (at 70, 160, 250, 350, and $500 \mu \mathrm{m})$. It provides complementary information of the spectral energy distribution in the regime where the thermal emission of cold and warm dust peak (between 10-500 K). ATLASGAL also has a substantial overlap with the Bolocam Galactic Plane Survey (BGPS, Aguirre et al. 2011) at $1.1 \mathrm{~mm}$ and partially overlaps with the JCMT SCUBA-2 survey of the Galactic plane (Di Francesco 2008) at $850 \mu \mathrm{m}$. There are common complexes also covered by the HOBYS program (Herschel imaging survey of OB young stellar objects; Motte et al. 2010). The Coordinated Radio and Infrared Survey for High-Mass Star Formation at $5 \mathrm{GHz}$ (CORNISH; Hoare et al. 2012; Purcell et al. 2013), and other surveys in the radio regime probe free-free emission of ionized gas surrounding OB type stars.

The combination of these surveys therefore provides a complete view of the spectral energy distribution (SED) from infrared to radio wavelengths, which is necessary to probe the nature of dust clumps. This reveals purely dust sources, which can be starless or prestellar, mid-infrared bright protostars that just started to heat up their surroundings, and massive stars where the ionizing emission leads to the development of UC-H II regions which then expand becoming optically visible H II regions. The dust emission at $870 \mu \mathrm{m}$ measured in the ATLASGAL survey is therefore sensitive to sources in all evolutionary stages with both cold and warm gas (Schuller et al. 2009; Urquhart et al. 2013b).

The ATLASGAL survey has been used to study various objects in specific environments in the Galaxy, and here we give an overview of them. For a sample of Galactic bubbles, identified from Spitzer-GLIMPSE data, Deharveng et al. (2010) use ATLASGAL to study the dense and cold material at the borders of H II regions. Beuther et al. (2012) uses ATLASGAL data to reveal the Galactic structure. In a limited range of Galactic longitude $\left(10^{\circ}<\ell<20^{\circ}\right)$, Tackenberg et al. (2012)

1 Observing runs: 078.F-9040, 181.C-0885 (ESO); 079.C-9501, 081.C-9501 (MPIfR); and Chilean data. 
use ATLASGAL to identify a population of starless clumps. The properties of dust clumps associated with various signposts of massive star-formation were determined in a series of papers. Urquhart et al. (2013a) studied the physical properties of sources from the Methanol-MultiBeam survey (MMB, Green et al. 2009), a sample of H II regions from the CORNISH survey (Urquhart et al. 2013b) and MYSOs from the RMS survey (Urquhart et al., in prep.). The projected image of dust lacks, however, any information on the line-of-sight distribution of the material, for which spectroscopic observations are required. Numerous molecular line follow-up observations have therefore been triggered by the ATLASGAL survey. The MALT90 project maps over 2000 ATLASGAL sources in 16 lines (Foster et al. 2011; Jackson et al. 2013). Wienen et al. (2012) presents an extensive follow-up campaign of the $\sim 1000$ brightest dust clumps in $\mathrm{NH}_{3}$ in order to determine kinematic distances and gas temperatures.

The first catalog of compact sources from the ATLASGAL survey is presented by Contreras et al. (2013) and focuses on a limited range in Galactic longitude $\left(-30^{\circ}<\ell<21^{\circ}\right)$. Here we aim to complement this work by covering the full area of the survey and at the same time specifically addressing the population of embedded, smaller size-scale objects (see Sect. 3.6) compared to Contreras et al. (2013).

The paper is organized as follows. Section 2 describes the data processing, and Sect. 3 presents the source extraction method and comparison with the previous catalog. Section 4 presents the catalog and the properties of the extracted sources. In Sect. 5 we compare the sources with mid-infrared diagnostics to estimate their fraction associated with ongoing star formation and study their statistical properties. Then we present their properties, such as the Galactic distribution and typical fluxes of quiescent and star-forming sources in Sect. 6. Based on these statistics we estimate a Galactic star formation rate and formation time scales in Sect. 7. We summarize the results in Sect. 8.

\section{Observation and data reduction}

This paper is based on all the data taken for the survey between 2007 and 2010. The observing strategy and the main steps of the data reduction procedure are described in detail by Schuller et al. (2009) and Contreras et al. (2013). The data were reduced with the $B o A$ software (Schuller 2012) $)^{2}$, and emission from larger scales was iteratively recovered until convergence was reached after 15 iterations.

The absolute position accuracy of the ATLASGAL survey is discussed in detail in Contreras et al. (2013). The astrometry of the dataset and the derived source positions are estimated to be accurate to the pointing accuracy of the telescope, which is $\sim 2-3^{\prime \prime}$. The absolute flux uncertainty is estimated to be less than $\sim 15 \%$ (Schuller et al. 2009). Variations in the sky emission ("sky-noise") mimic emission from extended astronomical objects. Ground-based bolometer arrays are therefore not well suited to measuring extended emission, since the emission from larger angular scales is removed when subtracting the correlated noise from the maps. The final emission maps of the survey are sensitive to angular scales up to 2.5 , thus the current data reduction is optimized to enhance compact sources. The final maps were gridded on three-by-three degree tiles with $\sim 4.5$ overlap between adjacent emission maps. The pixel size is $6^{\prime \prime}$ which is

\footnotetext{
2 http://www.eso.org/sci/activities/apexsv/labocasv. html
}

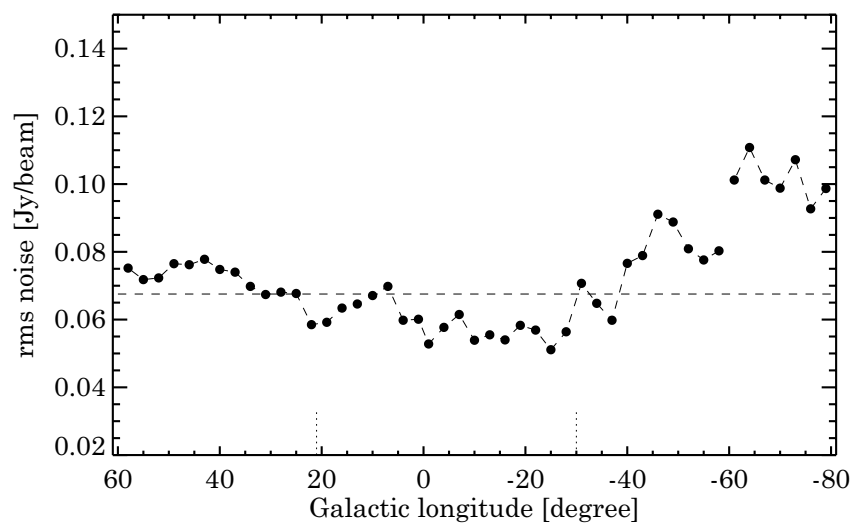

Fig. 1. rms noise level determined on the individual tiles of maps using a Gaussian fitting to the distribution of the pixel values between $|b| \leq 1^{\circ}$. The region analyzed in Contreras et al. (2013) is indicated by dotted lines. The horizontal dashed line shows the average $0.07 \mathrm{Jy} / \mathrm{beam}$ noise level for the main part of the survey $\left(|\ell| \leq 60^{\circ}\right)$.

about one third of the beam size. The data are publicly available at http: //atlasgal .mpifr-bonn.mpg.de/.

The average noise level was determined from the $b \leq 1^{\circ}$ portions of the maps where the vast majority of the emission originates and because the noise increases rapidly towards the edges of the maps due to a lower number of overlapping coverages. The noise was determined from a Gaussian fit to the distribution of the pixel values in the maps and is found to be on average $\sim 70 \mathrm{mJy} /$ beam over the whole survey region. The average noise varies $(\sim 20 \%)$ as a function of $\ell$ due to the nonhomogenous coverage of the observed regions and varying observing conditions (see Fig. 1). In the longitude ranges $\ell>40^{\circ}$ and $\ell<-40^{\circ}$ the average noise increases to $70-90 \mathrm{mJy} / \mathrm{beam}$, while the noise is $\sim 50-60 \mathrm{mJy} /$ beam in the central part of the plane. In the extension, between $-80^{\circ} \leq \ell \leq-60^{\circ}$, the noise is higher $(\sim 110 \mathrm{mJy} / \mathrm{beam})$ than in the main part of the survey due to fewer coverages and shorter observing time.

In the following we extract the population of compact sources in the Galactic plane using the whole ATLASGAL data set. However, due to the increased noise in the extension, we based our analysis only on the main part of the survey within the $|\ell| \leq \pm 60^{\circ}$ in Galactic longitude range. The identified compact objects mainly correspond to starless, precluster dense material, protostars, protoclusters, compact H II regions, as well as overdensities in the clumpy and filamentary cloud structures.

\section{Source extraction}

In the following we assume that the $870 \mu \mathrm{m}$ emission arises from thermal continuum emission from dust and that the contamination from free-free emission is negligible. The filter of LABOCA admits a $60 \mathrm{GHz}$ frequency range centered on $345 \mathrm{GHz}$ (Siringo et al. 2009), thus covering the CO (3-2) rotational transition at $345.796 \mathrm{GHz}$ and other lines. Here we do not account for any line contamination; nevertheless, as discussed in Schuller et al. (2009), the contribution to the derived fluxes may be more than the usual $15 \%$ flux calibration uncertainty, but only towards the most extreme sources, such as hot molecular cores, strong outflow sources, and bright photon-dominated regions, and is not likely to be a problem with the vast majority of the ATLASGAL sources.

\subsection{Overview of source extraction algorithms}

Systematic source identification algorithms have been developed to analyze ground-based millimeter/submillimeter maps, such as 
Clumpfind (Williams et al. 1994), and Gaussian source fitting in different implementations, originally developed by Stutzki $\&$ Güsten (1990). The arrival of fast-mapping capabilities by the PACS and SPIRE instruments onboard Herschel boosted the development of various source-extraction algorithms. The space-based observations provide the possibility for efficient mapping, but also the imaging of a significant amount of largescale emission, which cannot be recovered by ground-based surveys. Therefore several new methods have been developed to deal with the extended emission and also to handle multiwavelength datasets, such as those of Herschel (e.g., MRE-GCL, Multi-resolution and Gaussclumps algorithm, Motte et al. 2010; CuTeX: Molinari et al. 2011; Getsources: Men'shchikov et al. 2012). A detailed overview of these different source-extraction algorithms that discusses their advantages and disadvantages is given in Men'shchikov et al. (2012).

The majority of these algorithms assume a uniform noise distribution that is not realistic, especially for ground-based observations that may be done under different meteorological conditions, different elevations, etc. It is therefore unavoidable that large-area ground-based surveys show varying noise levels over the survey region. The SExtractor algorithm (Bertin \& Arnouts 1996) uses a $\sigma$-clipping method to handle the varying noise level and was originally developed for optical and infrared images to identify stars (point sources) and galaxies. However thermal emission from the spatially extended cloud structure usually shows a more complex morphology. For example, the population of compact sources is deeply embedded in dense clouds. Contreras et al. (2013) have successfully applied the SExtractor algorithm to a substantial part of the ATLASGAL survey $\left(-30^{\circ}<\ell<21^{\circ}\right)$. This method applied to dust emission maps pulls out properties of the whole clump because it is not optimized to separate compact emission from the more diffuse envelope. Therefore it can be considered to work similarly as a contouring algorithm like Clumpfind without any assumption on the source characteristics.

Another, in many ways complementary approach is to assume a certain characteristic or property for the embedded sources. The Gaussclumps algorithm (Stutzki \& Güsten 1990; Kramer et al. 1998) was originally developed to identify coherent structures of 3D molecular-line (position, position, velocity: ppv) data cubes, and it assumes a Gaussian intensity distribution to identify structures. The interstellar medium exhibits a clumpy morphology with the embedded sources on much smaller spatial scales superimposed on this background emission. Since our main interest is to identify these more compact sources, i.e. cores and clumps, we based their identification on the assumption that they exhibit a Gaussian intensity distribution. The assumption of a Gaussian intensity profile is adequate for compact sources with sizes of a few times the beam (e.g., Motte et al. 2007, see also Fig. A.1).

Our source extraction method follows the scheme originally developed in Motte et al. (1998) and presented in Motte et al. (2007), which is referred to as the MRE-GCL method. It has been used to identify compact sources of thermal dust emission by different groups for complex regions, such as W43 (Motte et al. 2003), Cygnus-X (Motte et al. 2007), and the NGC 6334-NGC 6357 complexes (Russeil et al. 2010). It has also been applied for smaller regions, such as RCW 106 (Mookerjea et al. 2004), NGC 2264 (Peretto et al. 2006), and G327.3-0.6 (Minier et al. 2009). The main steps are to first disentangle the compact sources from the more diffuse, extended emission of filamentary clouds. This is done by a multiscale wavelet transformation that is used to filter out the larger scale structures (see examples in Figs. 2, A.1) and then use the Gaussclumps algorithm to extract sources. These steps in the method are described in more detail in the following two sections, Sects. 3.2 and 3.3.

\subsection{Multiscale decomposition}

Molecular clouds exhibit structures at various scales, and part of their material is organized into large-scale filaments (e.g., André et al. 2010; Molinari et al. 2010a; Kainulainen et al. 2011). Although ground-based bolometers are insensitive to uniform diffuse emission, they recover a significant amount of extended structures of dust seen towards the Galactic plane. Since the ATLASGAL maps contain emission from 19.'2 up to 2.5 spatial scales, it is desirable to remove the extended emission in order to extract the properties of embedded sources.

At distances up to $1 \mathrm{kpc}$, the spatial resolution of the survey corresponds to $<0.1 \mathrm{pc}$ physical scales, thus to rather individual cores. Placed at a larger distance beyond $10 \mathrm{kpc}$, this translates to $>1$ pc size-scale objects, therefore corresponding to cloud structures. As opposed to single complexes, the choice of a physical scale is not trivial since the whole Galaxy is seen in projection along the line-of-sight.

We therefore visually examined the structures at different scales aiming to identify the best spherically symmetric, centrally condensed compact objects. This way we chose to optimize the extraction method to be sensitive to structures with angular scales from the 19.2 resolution element to $\sim 50^{\prime \prime}$, which translate to a physical scale of $0.4-1 \mathrm{pc}$ at $4 \mathrm{kpc}$. This is also a reasonable physical scale for compact structures when assuming that a large fraction of the sources lie at the typical distance of $\sim 4 \mathrm{kpc}$ (e.g., Wienen et al. 2012), while Peretto \& Fuller (2010) finds that $95 \%$ of the IRDCs are at distances of $<6 \mathrm{kpc}$ with the mean distance between $3 \mathrm{kpc}$ and $5 \mathrm{kpc}$. Here we aim therefore to extract the properties of compact sources with typical size scales of 0.4 pc to $1 \mathrm{pc}$, commonly denoted as clumps (e.g., Bergin \& Tafalla 2007; Motte \& Hennebelle 2009).

Since our primary interest is in these embedded sources, we systematically remove emission from cloud structures on larger scales that we consider as extended emission originating from the embedding cloud. To do this we decomposed the emission into different spatial scales using a wavelet transformation with the method of Starck \& Murtagh (2006). Emission from smaller than twice the maximum required $50^{\prime \prime}$ scale was then summed up. Owing to the wavelet decomposition, negative artifacts appear around bright sources, and we have set these negative artifacts to zero to help the convergence of the algorithm (see Kramer et al. 1998; Motte et al. 2007). The emission with different scales is illustrated in Fig. 2 (for an isolated source, see Fig. A.1).

\subsection{Extraction of compact sources - Gaussclumps}

Although Gaussclumps does not deal with varying noise levels, it is very important to consider the non-uniform noise distribution of the maps to avoid detecting spurious sources and missing genuine sources in a region with lower noise. To overcome this, we used the weight maps and calculated from pixel to pixel signal-to-noise maps using the formula: $S / N=$ flux $\times \sqrt{\text { weight, }}$ where flux corresponds to the flux density in the emission maps, and the weight is computed when combining signals of all bolometers into a map. The weight is related to the noise as $1 / \mathrm{rms}^{2}$ where the rms is the standard deviation of the signals of the bolometers (see Schuller et al. 2009, for more details). 

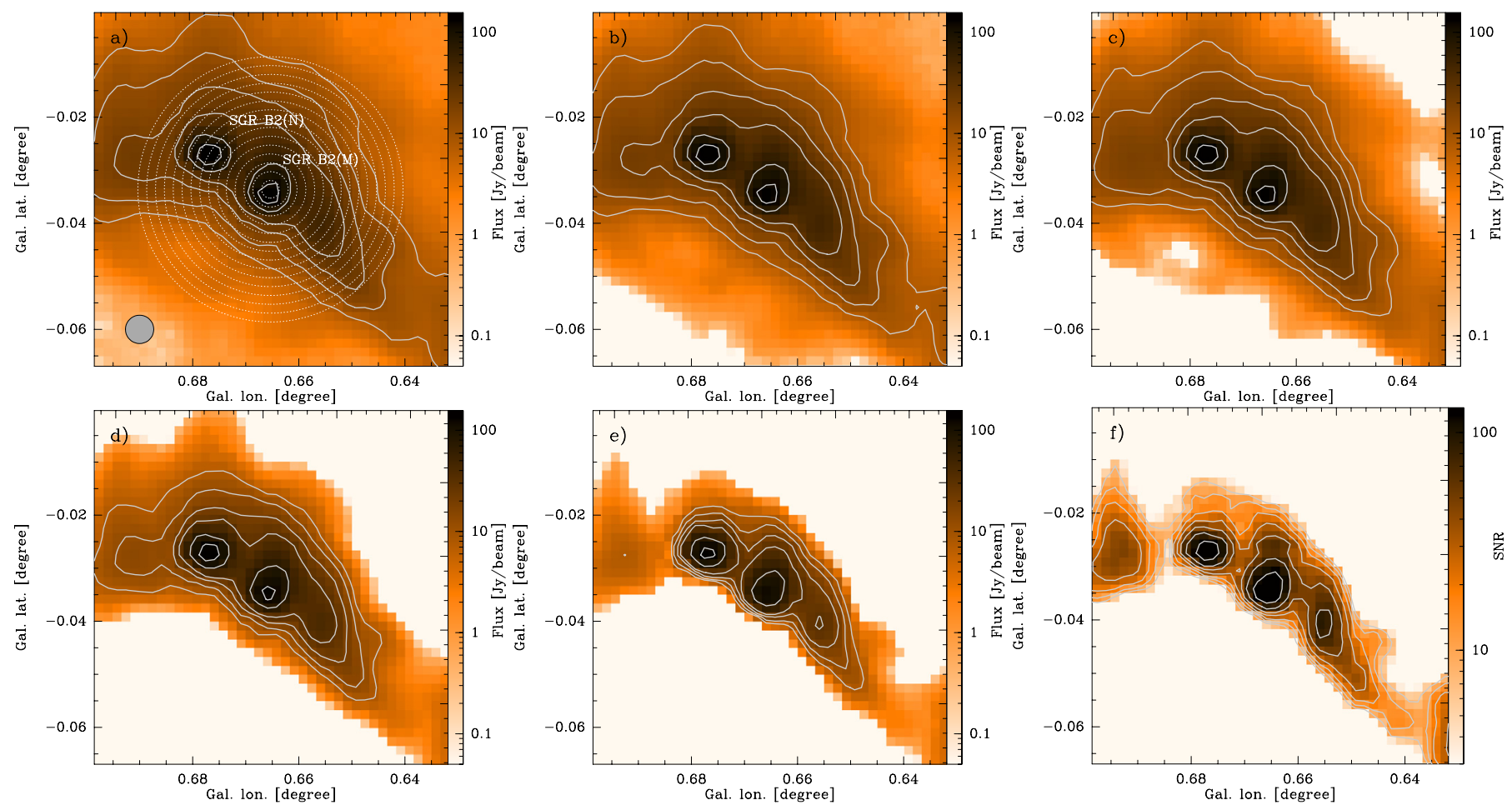

Fig. 2. Example of the multiscale decomposition towards the brightest region at $870 \mu \mathrm{m}$ in the whole survey, which hosts SgrB2(N) and SgrB2(M). The impact of flux loss due to filtering is the most severe towards this region. Contours start at $7.5 \mathrm{Jy} / \mathrm{beam}$ and increase on a logarithmic scale to $120 \mathrm{Jy} /$ beam. Dotted circles mark the regions where the radial averaging has been done (see Sect. 3.3 and Fig. 5 for details). The beam size of $19.2^{\prime \prime}$ is shown in panel a). Panels a) to e) show maps with different scales of filtering: from no filtering to maps where background emission is increasingly removed. The catalog is generated from a filtering level corresponding to the e) panel, where emission until $2 \times 50^{\prime \prime}$ scales is summed up. The peak flux density of the object in this case decreases by $20 \%$ from the original images to the most compact one, while the size decreases by only $10 \%$. The f) panel shows the filtered $\mathrm{S} / \mathrm{N}$ map used as the input for the source extraction algorithm containing the same spatial scales as the e) panel. The scaling is logarithmic between $3-120 \sigma$, contours start at $7 \sigma$ increasing on a logarithmic scale.

The intensity of the weight maps exhibits variations of $\sim 10 \%$ on a $60^{\prime \prime}$ scale, so the spatial variations are smaller than the expected size of compact sources. Therefore we use the $\mathrm{S} / \mathrm{N}$ maps as the input files for the source extraction method, and use Gaussclumps with a threshold of $\sim 5$, which corresponds to $5 \times$ the local $\mathrm{rms}^{3}$. The choice of this threshold is justified in Sect. 3.4.

The position and size of the sources were derived from the output of Gaussclumps, while the peak flux values were computed using the flux values from the $\mathrm{S} / \mathrm{N}$ maps multiplied by the noise level measured on the weight maps.

Since we apply this procedure for each tile, and there is a $\sim 4.5^{\prime}$ overlap between the individual ATLASGAL tiles, sources falling on these edges are found twice by the algorithm. We eliminated these duplicates by systematically keeping those detections that are closer to the center of the map, in which they are identified. After rejecting duplicate sources, we also rejected sources with sizes smaller than the beam as these are likely to correspond to noise peaks in the maps (see e.g., Kramer et al. 1998).

As a sanity check we visually inspected all sources with outlying (i.e., the highest and lowest) fluxes and aspect ratios and found them to be genuine sources. We discuss the impact of the

\footnotetext{
3 The input parameters for Gaussclumps were an initial guess for the size of $1.5 \times$ the beam FWHM, and the stiffness parameters $\left(s_{0}, s_{a}, s_{c}\right)$ have been set to 1 . These control the weighting of the Gaussian function in order to fit a peak intensity and position close to the observed value. For a detailed description of these parameters we refer to Stutzki \& Güsten (1990) and Kramer et al. (1998).
}

filtering on the extracted source parameters in Appendices A.1 and A.2.

All of the survey region was inspected for missed sources and we found that visible but undetected structures fall below the $\mathrm{S} / \mathrm{N}$ threshold used for the extraction.

\subsection{Completeness limit}

We estimate the completeness limit of our source extraction method by adding artificial sources to the input maps of Gaussclumps. Using the same input parameters as for the cata$\log$, we measure the number of extracted artificial sources as a function of peak flux, following a similar procedure to the one described by Contreras et al. (2013). For these tests we used a simulated field of $20 \times 9$ sq. degree size, which corresponds to 20 ATLASGAL tiles and injected 9989 artificial sources. This is similar to the total number of sources we find for the full survey. To mimic a uniform background noise, the first set of artificial sources were injected on maps with a normal distribution of noise values with a standard deviation of $\sigma=60 \mathrm{mJy} / \mathrm{beam}$. The injected sources have peak flux densities following a uniform distribution up to $1.5 \mathrm{Jy} / \mathrm{beam}$, with $30^{\prime \prime}$ FWHM size, an aspect ratio of 1 and a Gaussian flux distribution in order to imitate spherically structured cores.

The second test was made with the same characteristics as for the artificial sources, but injected on a background map with a varying noise level. For this we used a map with real observations, but smoothed with a Gaussian kernel of 31 by 31 pixel array, which has a FWHM of five-by-five pixels in order to imitate 


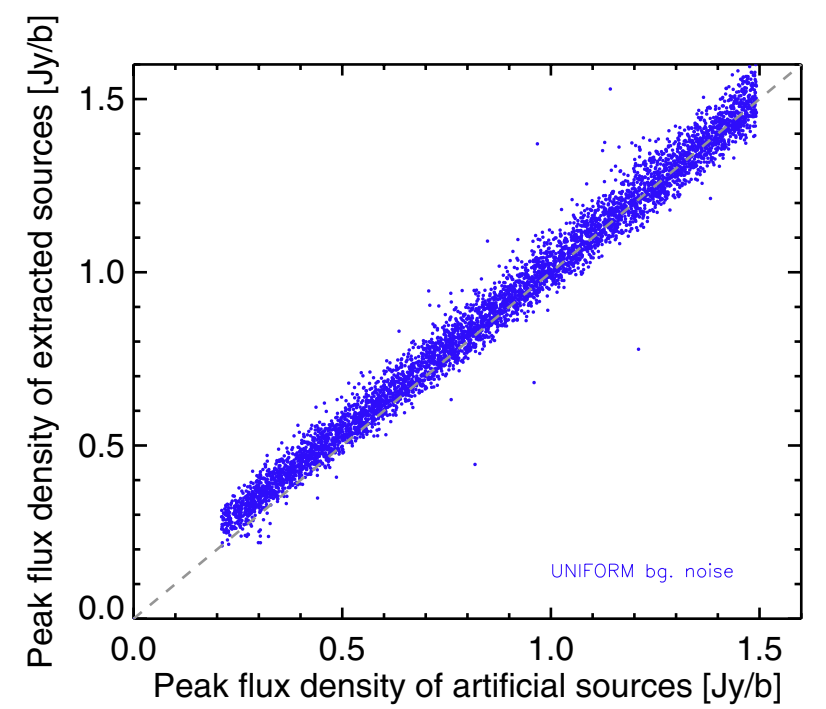

Fig. 3. Comparison between the peak flux densities of the extracted and the artificial sources. For clarity, only the uniform noise simulations are shown, but the result obtained from the other two tests are similar.

the extended emission from molecular clouds. A third test was done using the maps with varying background noise and the same filtering level as for the full catalog. We ran our extraction algorithm down to a fixed threshold of $\sim 3 \sigma$ and therefore extracted sources down to the $0.2 \mathrm{Jy} /$ beam. This threshold was chosen to be lower than the one used for the source extraction on the real data in order to explore the lower limits of our completeness.

As a consistency check, we compared the fluxes of the injected and recovered sources and find good agreement between these values for all three simulations. Figure 3 shows the comparison between the injected and extracted peak flux densities for the case of normal noise distribution. In all of the simulations, we find that the measured source properties lie close to unity compared to the injected parameters for high peak flux densities. Close to the detection threshold, we see a deviation from unity with the measured fluxes being offset to slightly higher than the injected values. This offset is at most $1 \sigma(60 \mathrm{mJy})$ in the peak flux densities introducing $<20 \%$ error in the peak fluxes for the weakest sources and is a result of flux boosting due to the constructive interference with the noise that determines the fitting of the peak flux.

Figure 4 shows the detection ratio of the sources as a function of peak flux density, where we allowed a positional offset of $10^{\prime \prime}$ between the artificial and the extracted sources. We find that our catalog is complete to $99.4 \%$ above $7 \sigma$ and $97 \%$ of the injected sources are recovered at $5 \sigma$. This was therefore chosen as a detection threshold for the extraction for the full survey. A lower value would result in a less reliable catalog, and the completeness level at lower flux densities would also be limited, which is not desirable for statistical studies. We stress that below a $7 \sigma$ noise level, measured on the input maps, our catalog is not complete, and genuine sources may be missed. Our completeness level is comparable to those of other source extraction methods, such as the one used by Contreras et al. (2013).

\subsection{Physical parameters of the compact sources}

Since the sources are identified and extracted from maps where the extended emission was removed, the corresponding physical parameters, such as the size and the peak flux, are different compared to the original emission maps. These parameters are

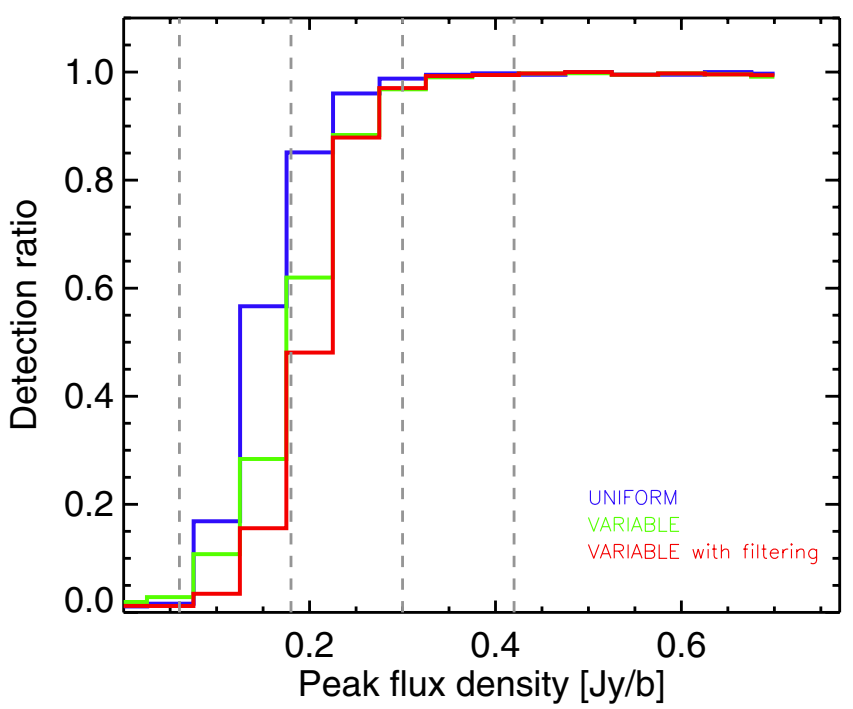

Fig. 4. Detection ratio of recovered versus injected sources shown as a function of peak flux. Blue, green, and red line show the uniform and the varying noise extraction without and with filtering, respectively. The filtering corresponds to the $100^{\prime \prime}$ filtering level we used to produce the catalog. Gray dashed lines correspond to $1 \sigma, 3 \sigma, 5 \sigma$, and $7 \sigma$ noise levels.

more likely to reflect the real properties of the embedded compact sources as illustrated in Fig. 2 (another example is shown in Fig. A.1 for an isolated source) and in Fig. 5, where we show the azimuthally averaged intensity profile from the original and the filtered maps used for the source extraction. It illustrates that by removing the extended emission, the measurement of the Gaussian parameters of the embedded source improves. We have tested the impact of the multiscale decomposition on the measured fluxes and sizes by extracting the sources using different scales. We found that the impact on the measured size is in most cases very small, because the majority of the sources are only marginally resolved, and their morphology is dominated by compact emission. For the peak flux values we find that the difference is less than $20 \%$ for isolated sources, however in complex regions, especially for bright, massive clouds, the extended emission contributes more to the total flux. This can be as high as $50 \%$ of the total flux in such regions (see also Appendix A for more details).

We measure the physical parameters of the sources using the formulae from Schuller et al. (2009) and assuming optically thin emission of dust at $870 \mu \mathrm{m}$. The column density is derived from

$N\left(\mathrm{H}_{2}\right)=\frac{F_{v} R}{B_{v}\left(T_{\mathrm{d}}\right) \Omega \kappa_{v} \mu_{\mathrm{H}_{2}} m_{\mathrm{H}}}$,

where $F_{v}$ is the peak flux density, $\Omega$ the beam solid angle, $\mu_{\mathrm{H}_{2}}$ the mean molecular weight of the interstellar medium with respect to hydrogen molecules, which is equal to 2.8 (Kauffmann et al. 2008) $)^{4}$, and $m_{\mathrm{H}}$ is the mass of an hydrogen atom. We adopt here the same assumptions as Schuller et al. (2009): a gas-to-dust mass ratio of 100 and $\kappa_{v}=1.85 \mathrm{~cm}^{2} \mathrm{~g}^{-1}$, which is interpolated to $870 \mu \mathrm{m}$ from Table 1, Col. 5 of Ossenkopf \& Henning (1994). At our completeness level of $7 \sigma$, we are thus sensitive to column

\footnotetext{
4 The mean molecular weight is commonly defined with respect to free particles in the gas, in which case $\mu=2.33$ and the derived column density reflects the number density of free particles. Using $\mu_{\mathrm{H}_{2}}=2.8$ gives the column density of $\mathrm{H}_{2}$ molecules, and one has to multiply our $N\left(\mathrm{H}_{2}\right)$ values by a factor of $\sim 1.2$ to be consistent with other studies using $\mu=2.33$.
} 
T. Csengeri et al.: ATLASGAL - a catalog of dust condensations in the Galactic plane

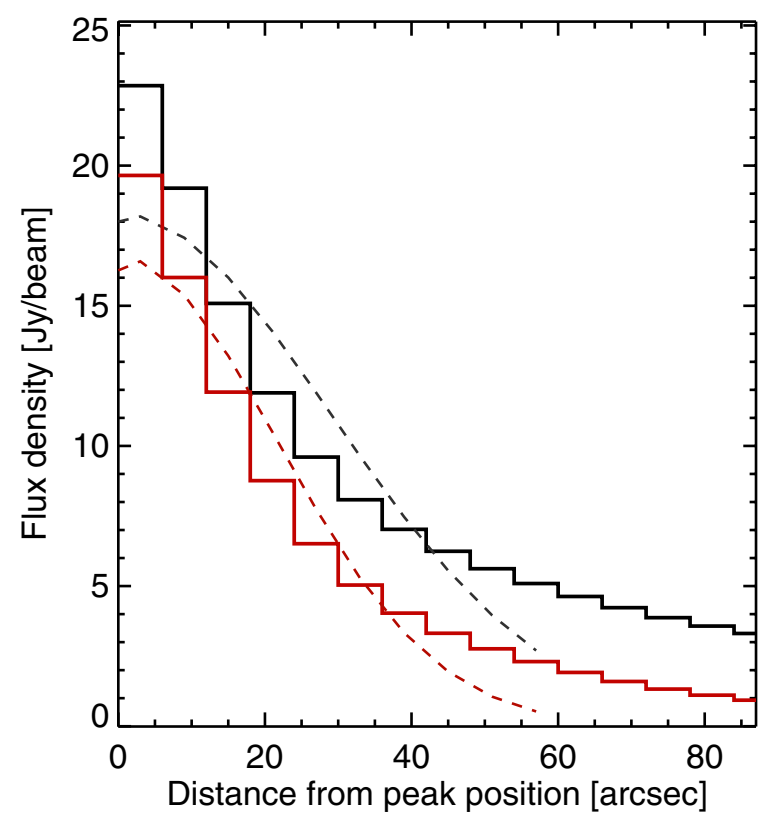

Fig. 5. Azimuthally averaged emission profile averaged over the 100 brightest sources. The black line corresponds to the original images, while the red line shows the images filtered above the $100^{\prime \prime}$ scale. Dashed lines show the Gaussian fits to these profiles.

densities between $\sim 7 \times 10^{21}-4 \times 10^{22} \mathrm{~cm}^{-2}$ for warm $\left(T_{\mathrm{d}}=30 \mathrm{~K}\right)$ and cold $\left(T_{\mathrm{d}}=10 \mathrm{~K}\right)$ gas, respectively. We estimate the mass as:

$M=\frac{S_{v} R d^{2}}{B_{v}\left(T_{\mathrm{d}}\right) \kappa_{v}}$

where $S_{v}$ is the integrated flux and $d$ corresponds to the distance of the sources. For a typical dust temperature of $T_{\mathrm{d}}=15 \mathrm{~K}$, our completeness limit corresponds to $\sim 270 M_{\odot}$ at $8 \mathrm{kpc}$ and $1 M_{\odot}$ at $0.5 \mathrm{kpc}$. The ATLASGAL survey therefore probes all massive clumps in the inner Galaxy and in the $<1 \mathrm{kpc}$ nearby regions intermediate-mass cores as well.

Since with ATLASGAL we probe the whole inner Galaxy, it is crucial to assess what fraction of the extracted sources may sustain high-mass star formation. Motte et al. (2007) adopted a limit of $40 M_{\odot}$ for defining massive dense cores (MDCs) based on an unbiased study of the high-mass star-forming complex, Cygnus- $X$. The sources were found to have an average size of $0.13 \mathrm{pc}$, and the mass was calculated assuming a density profile of $\rho(r) \propto r^{-2}$. Using high angular-resolution observations these MDCs have been confirmed to host individual high-mass protostars (Bontemps et al. 2010; Csengeri et al. 2011b). We extrapolate this definition to our average spatial resolution of $0.4 \mathrm{pc}$ (see Sect. 3.2), and find that the same $\rho(r) \propto r^{-2}$ density profile corresponds to minimum mass of $\sim 125 M_{\odot}$. However, as observed in Cygnus- $\mathrm{X}$, it is very likely that MDCs are embedded in massive clumps on the $0.4 \mathrm{pc}$ scale of our resolution. For example, the massive clump of DR2 $1(\mathrm{OH})$ with $0.6 \mathrm{pc}$ size is fragmented into three MDCs on a $0.13 \mathrm{pc}$ scale. Each of these MDCs were shown to host individual high-mass protostars (Csengeri et al. 2011b). If the ATLASGAL clumps fragment on smaller than $0.4 \mathrm{pc}$ scale, they should exhibit a shallower density profile. Therefore we estimate a minimum mass range between the $\rho(r) \propto r^{-2}$ density profile (extreme lower limit, $\sim 125 M_{\odot}$ ) and a uniform density profile (extreme upper limit, $\sim 1150 M_{\odot}$ ). We adopt here an average value of these two limiting cases of $650 M_{\odot}$ as an approximate threshold for ATLASGAL clumps likely to host MDCs and high-mass protostars. This is consistent with other studies similarly suggesting that clumps below $1000 M_{\odot}$ still have the potential to form massive stars as long as they are compact (e.g., Lada \& Lada 2003; Urquhart et al. 2013a) ${ }^{5}$.

\subsection{Comparison to previous work}

The source identification method described in Contreras et al. (2013) has been extended to the full ATLASGAL survey (Urquhart et al., in prep.) and here we give a comparison of the two methods.

As a first step we checked what fraction of our sources coincide with the source masks from Contreras et al. (2013) to compare the efficiency of identifying sources between the two methods. We find 42 sources in all that are identified in the current catalog, but were not found by SExtractor, and there are another 350 sources that were identified by SExtractor, but later rejected due to having a smaller number of pixels than the area of the beam. We visually inspected all of these sources and found them to be genuine sources.

We find 2508 sources identified by SExtractor, but not identified with the current method. This is partly because Contreras et al. (2013) lists sources to a lower, $3 \sigma$ threshold, and therefore the majority of these sources are primarily weak and fall below our $5 \sigma$ detection limit. A fraction of them belong to structures that are removed by the filtering we apply here. Sources with peak fluxes above our detection threshold were individually inspected and found to be part of cloud structures that are decomposed in a different way by our method.

As pointed out in Sect.3.1, the SExtractor software was originally developed for optical and infrared images to find stars and galaxies, which show lower levels of extended background emission than does the dust continuum from molecular clouds. Differences between the two catalogs come from the fact that the MRE-GCL method is more efficient at identifying small and compact sources, while SExtractor is better suited to finding larger sources. The two catalogs are therefore complementary in terms of source characteristics.

\section{Compact sources in the Galactic plane}

Here we present the result of the source extraction method described above. In total we have found 10565 sources in the $-60^{\circ} \leq \ell \leq+60^{\circ}$ longitude and $-1.5^{\circ} \leq b \leq+1.5^{\circ}$ latitude ranges, covering the Ist and IVth Galactic quadrants. Table 1 presents a sample of the compact source catalog, and the full catalog is available in electronic form. The table structure is as follows: column (1) corresponds to the source ID, then we give the source name (2) with the position in galactic coordinates and in J2000 equatorial coordinates $(3,4)$ (in hexagesimal format). The physical parameters of the sources follow: the beam-convolved major $\left(\Theta_{\text {maj }}\right)$ and minor axes $\left(\Theta_{\text {min }}\right)$ in arcseconds $(5,6)$, the position angle of the fitted Gaussian measured from north to east (7), the average FWHM source size

5 Our criterion for clumps to form massive stars is more selective compared to Tackenberg et al. (2012), who adopt a mass limit of $1000 M_{\odot}$. They select on average larger structures with larger integrated fluxes. Our analysis yields on average $6 \times$ lower masses owing to the different source identification and a different $\kappa_{v}$. Consequently, their mass limit corresponds to $1000 M_{\odot} / 6 \simeq 170 M_{\odot}$ in our analysis. Our criterion for clumps forming massive stars is therefore four times more conservative. 
Table 1. Dust condensations identified in the ATLASGAL survey.

\begin{tabular}{|c|c|c|c|c|c|c|c|c|c|c|}
\hline ID & Name & $\begin{array}{c}\text { RA } \\
{[\mathrm{J} 2000]}\end{array}$ & $\begin{array}{c}\text { Dec } \\
{[\mathrm{J} 2000]}\end{array}$ & $\begin{array}{c}\Theta_{\text {maj }} \\
{[\operatorname{arcsec}]}\end{array}$ & $\begin{array}{c}\Theta_{\min } \\
{[\operatorname{arcsec}]}\end{array}$ & $\begin{array}{l}\text { PA } \\
{\left[{ }^{\circ}\right]}\end{array}$ & $\begin{array}{l}F W H M \\
\text { [arcsec] }\end{array}$ & $\begin{array}{c}F_{v} \\
{[\mathrm{Jy} / \text { beam }]}\end{array}$ & $\begin{array}{c}S_{v} \\
\text { [Jy] }\end{array}$ & $S / N$ \\
\hline 1 & G300.1627-0.0899 & $12: 27: 08.6$ & $-62: 49: 51.6$ & 33 & 23 & 97 & 28 & 1.18 & 2.51 & 12.44 \\
\hline 2 & G300.2169-0.1106 & $12: 27: 35.9$ & $-62: 51: 24.2$ & 31 & 26 & 21 & 29 & 1.04 & 2.40 & 11.14 \\
\hline 3 & G300.5038-0.1763 & $12: 30: 03.5$ & $-62: 56: 50.4$ & 25 & 24 & 88 & 24 & 3.31 & 5.56 & 32.88 \\
\hline 4 & G300.8250+1.1517 & $12: 33: 40.9$ & $-61: 38: 51.0$ & 32 & 25 & 42 & 28 & 1.52 & 3.43 & 12.26 \\
\hline 5 & G300.9097+0.8811 & $12: 34: 14.5$ & $-61: 55: 23.4$ & 39 & 29 & 28 & 34 & 1.36 & 4.32 & 11.63 \\
\hline 6 & G300.9688+1.1456 & $12: 34: 53.2$ & $-61: 39: 47.3$ & 32 & 28 & 125 & 30 & 6.54 & 16.16 & 53.74 \\
\hline 7 & G301.0140+1.1137 & $12: 35: 15.0$ & $-61: 41: 52.2$ & 32 & 25 & 59 & 29 & 1.09 & 2.51 & 10.47 \\
\hline 8 & G301.1164+0.9596 & $12: 36: 02.1$ & $-61: 51: 28.6$ & 51 & 34 & -21 & 41 & 1.61 & 7.71 & 13.46 \\
\hline 9 & G301.1169+0.9771 & $12: 36: 02.8$ & $-61: 50: 25.9$ & 40 & 33 & 80 & 36 & 1.61 & 5.89 & 14.69 \\
\hline 10 & G301.1365-0.2256 & $12: 35: 35.2$ & $-63: 02: 31.9$ & 26 & 22 & 102 & 24 & 21.43 & 34.55 & 120.43 \\
\hline 11 & G301.1385+1.0092 & $12: 36: 14.7$ & $-61: 48: 35.0$ & 48 & 33 & 127 & 40 & 1.06 & 4.68 & 10.01 \\
\hline 12 & G301.6798+0.2456 & $12: 40: 33.1$ & $-62: 35: 59.1$ & 28 & 24 & 81 & 26 & 1.41 & 2.59 & 11.77 \\
\hline 13 & G301.7313+1.1038 & $12: 41: 17.6$ & $-61: 44: 39.6$ & 39 & 31 & 157 & 35 & 2.07 & 6.89 & 18.53 \\
\hline 14 & G301.7414+1.1013 & $12: 41: 22.6$ & $-61: 44: 50.4$ & 38 & 28 & 128 & 33 & 1.29 & 3.90 & 12.53 \\
\hline 15 & G301.8138+0.7811 & $12: 41: 53.3$ & $-62: 04: 12.1$ & 27 & 26 & 44 & 26 & 1.46 & 2.80 & 11.38 \\
\hline 16 & G302.0208+0.2517 & $12: 43: 31.0$ & $-62: 36: 22.0$ & 35 & 25 & 15 & 30 & 2.06 & 5.14 & 17.54 \\
\hline 17 & G302.0318-0.0607 & $12: 43: 31.7$ & $-62: 55: 07.4$ & 30 & 27 & 18 & 28 & 2.62 & 5.95 & 24.47 \\
\hline 18 & G302.0327+0.6254 & $12: 43: 43.0$ & $-62: 13: 58.6$ & 27 & 21 & 65 & 24 & 1.49 & 2.39 & 14.40 \\
\hline 19 & G302.3912+0.2804 & $12: 46: 44.4$ & $-62: 35: 11.3$ & 37 & 27 & -28 & 32 & 2.12 & 6.08 & 22.71 \\
\hline 20 & G302.4861-0.0310 & $12: 47: 31.4$ & $-62: 53: 58.0$ & 30 & 25 & 74 & 27 & 1.89 & 3.96 & 17.65 \\
\hline
\end{tabular}

Notes. The full table is available only in electronic form at the CDS and at http://atlasgal .mpifr-bonn.mpg.de

(beam-convolved $)^{6}(8)$, the peak flux (9), and the integrated flux (10) calculated assuming a 2D-Gaussian shape for the sources: $S_{v}=F_{v} \times\left(F W H M / F W H M_{\mathrm{bm}}\right)^{2}$, where $F_{v}$ is the peak flux, $F W H M$ is the geometric size of the source, and $F W H M_{\mathrm{bm}}$ is 19 '.2, the $\mathrm{S} / \mathrm{N}$ determined from the weight maps (11).

As an example we show the identified sources in one of the most complex regions in the Galaxy in Fig. 6. The top panel shows the large-scale emission of the W51 complex with a zoom on the most active site of star formation associated with W51 Main (e.g. Gaume et al. 1993). The bottom panel shows the filtered image that was used for the source extraction. This illustrates how the algorithm performs in complex environments: the filtering clearly removes large-scale emission, while all bright sources are recovered in the filtered maps.

We identified 296 sources in the extension between $280^{\circ} \leq$ $\ell \leq 300^{\circ}$ longitude and $-2.0^{\circ} \leq b \leq 1.0^{\circ}$ latitude range towards the Carina arm.

\subsection{Flux distribution}

We show the distribution of the peak flux densities in Fig. 7 with a linear least-square fit to the flux bins above the completeness limit and determine a slope of $N / \Delta F_{v} \sim F_{v}^{\alpha}$, where $\alpha=-1.44 \pm 0.03$. As a comparison, we plot the sources of Contreras et al. (2013) in blue, which shows an identical slope of $\alpha_{\text {Cont }}=-1.47 \pm 0.04$. Despite the differences of the extraction methods, the two distributions compare well, and we find the derived slopes to be consistent.

The distribution of the integrated intensity is shown in Fig. 8. Here we find larger differences between the results obtained by Contreras et al. (2013) and our method. We systematically derive lower integrated intensities for the individual sources compared to Contreras et al. (2013), and also we find fewer sources with large integrated flux. This illustrates the differences in the concept of the two catalogs, also discussed in Sect.3.6. Our

\footnotetext{
6 We determine the geometric average source size from the geometrical mean of the Gaussian axes: $F W H M=\sqrt{\Theta_{\text {maj }} \times \Theta_{\min }}$.
}

method is better at identifying centrally condensed, more compact objects, while Contreras et al. (2013) extracts more irregular and diffuse structures of clumps and small clouds. The observed shift in the distribution of integrated flux density is simply due to a combined effect of smaller peak fluxes derived for the individual sources (due to more efficient removal of the background emission) and smaller size scales (see also Sect.4.2) compared to Contreras et al. (2013).

We calculated the differential flux density distribution $\left(\Delta N / \Delta F_{v}\right)$ which has a slope of $-2.35 \pm 0.05$. This compares well with the value of -2.4 obtained from the BGPS cata$\log$ (Rosolowsky et al. 2010) and -2.2 for the slope of the clump mass function measured by Tackenberg et al. (2012) for a more limited sample of ATLASGAL sources extracted with the Clumpfind algorithm.

\subsection{Sizes and aspect ratios}

The distribution of the FWHM of the geometric sizes peaks at $\sim 25^{\prime \prime}$ as shown in Fig. 9 (top panel), suggesting that most of the sources are resolved by our 19.'2 beam. Although we measure source sizes up to 55", the majority of the sources exhibit compact characteristics with sizes of $<35^{\prime \prime}$, and with a median of $27^{\prime \prime}$.

In the lower panel of Fig. 9 we present a plot showing the aspect ratio distribution of the sample. This has a mean of 1.5 with a median value of 1.4 and decreases with increasing peak flux. For example considering sources above $5 \mathrm{Jy} /$ beam peak flux density, the mean aspect ratio drops to 1.3 with a median of 1.2, while the Contreras et al. (2013) sources have a median of 1.5 for these brightest sources. Again, our extraction method was optimized for compact dust condensations. The brightest sources generally exhibit a rather spherical morphology. Elongated sources are more likely to be inhomogeneities in the cloud structure mimicking embedded sources or simply the blend of several sources. 
T. Csengeri et al.: ATLASGAL - a catalog of dust condensations in the Galactic plane

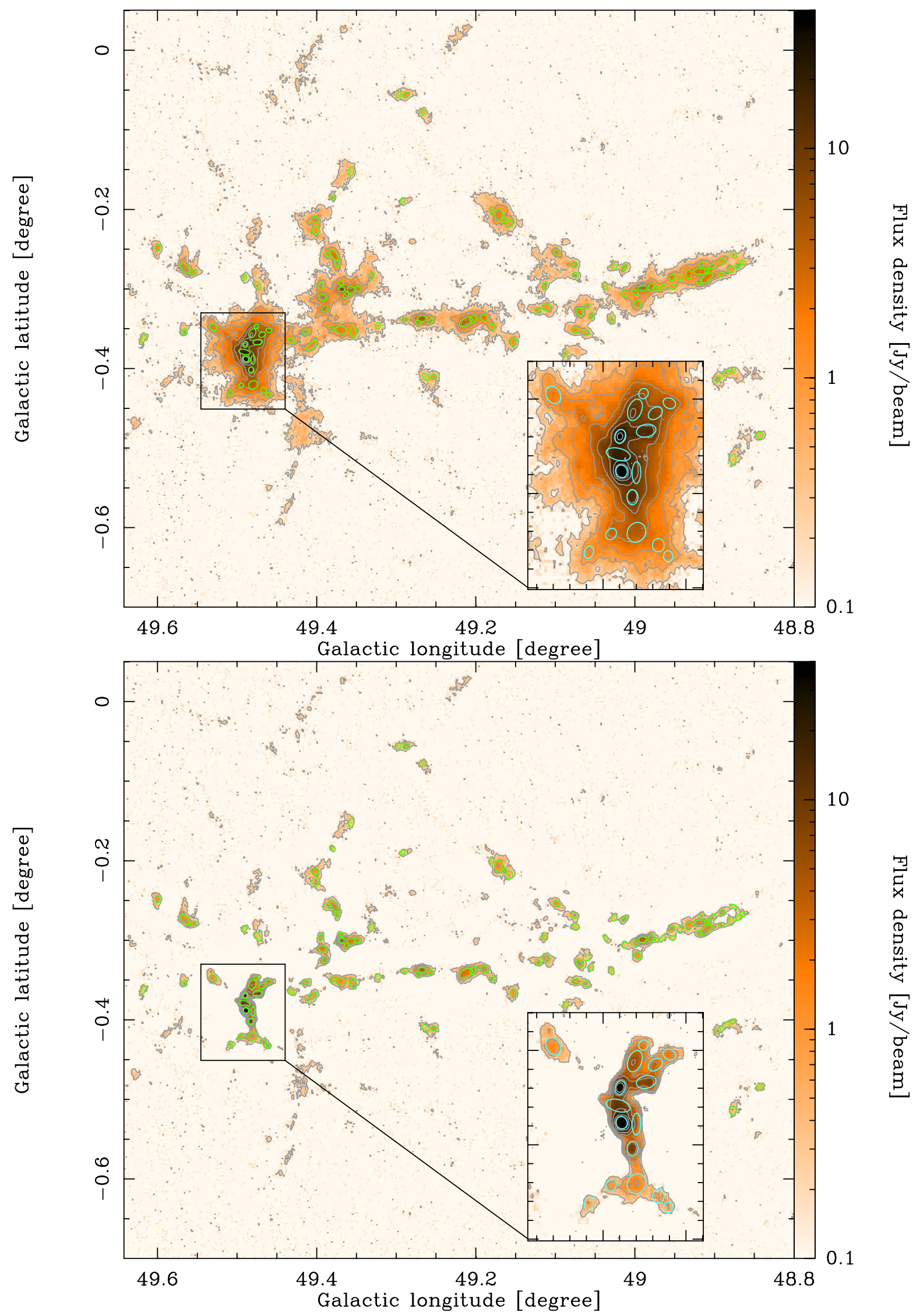

Fig. 6. Top: slice of the ATLASGAL survey showing the active star-forming region, W51, which contains both diffuse emission and compact sources. Color scale starts at a $0.1 \mathrm{Jy} / \mathrm{beam}$ and goes to $40 \mathrm{Jy} / \mathrm{beam}$. Dark gray contours start from $3 \sigma$ level $(0.21 \mathrm{Jy} / \mathrm{beam})$ and continue show $10 \sigma$, $20 \sigma, 40 \sigma, 80 \sigma, 160 \sigma, 320 \sigma$, and $500 \sigma$. Green circles show the position of the extracted sources. A zoom towards the brightest continuum sources is shown in the inset, also known as W51 Main. Bottom: the same region as in the top panel but with emission larger than 100" scale removed. 


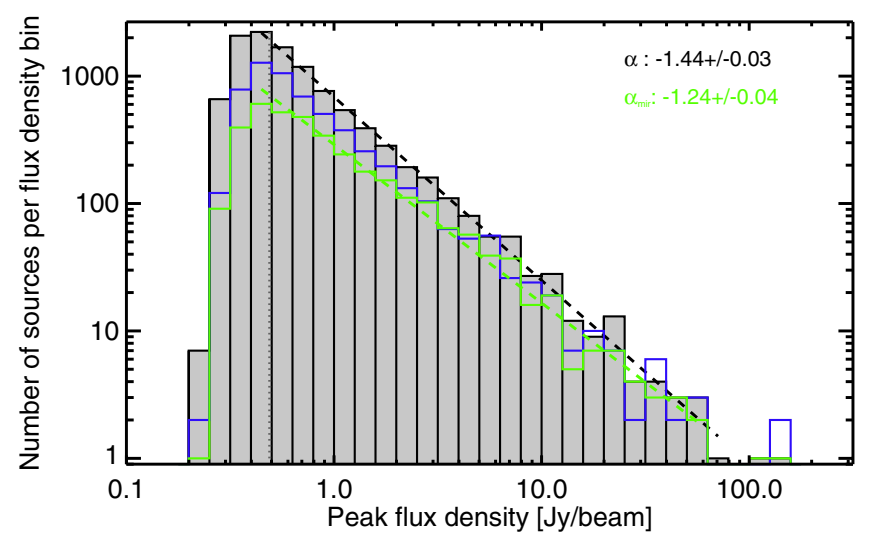

Fig. 7. Distribution of the peak flux density of the detected sources. The ATLASGAL sources from the current paper are shown in the black histogram with gray. The blue line shows the sources from Contreras et al. (2013). The black dashed line shows the measured slope of the distribution, and green indicates the star-forming ATLASGAL clumps (see Sect. 5 for details). A black dotted line corresponds to our $7 \sigma$ completeness limit when using an average of $70 \mathrm{mJy} / \mathrm{beam}$ noise level. The fits to the slope of the distributions are shown in dashed colored lines. We note that here we show the source count per bin, while in Rosolowsky et al. (2010) and Contreras et al. (2013) the differential source count, $\Delta N / \Delta F_{v}$ is shown.

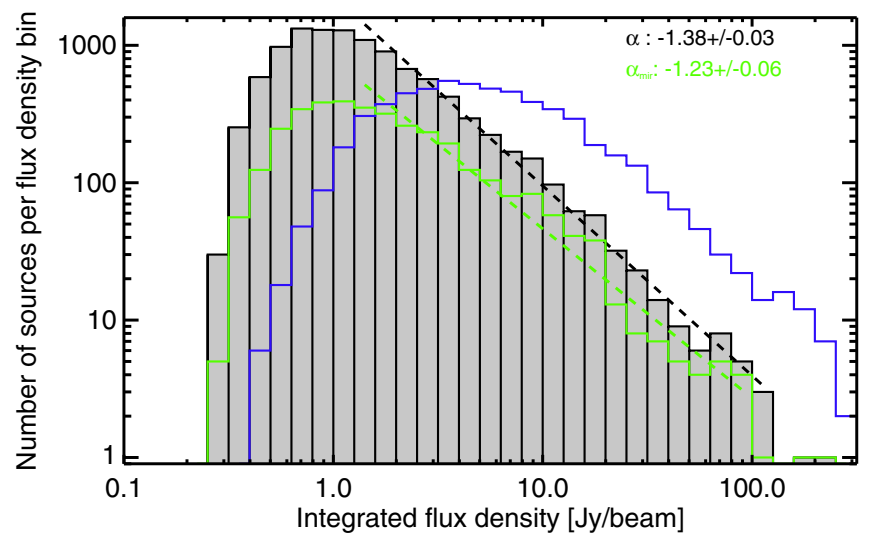

Fig. 8. Distribution of the integrated flux density of the detected sources. The black dashed line shows the measured slope of the distribution, green is the same distribution for the star-forming ATLASGAL clumps (see Sect. 5 for details). The blue histogram shows the sources from Contreras et al. (2013).

\section{Mid-infrared diagnostics to characterize star formation activity}

Mid-infrared emission traces warm dust, generally heated by embedded protostars, (M)YSOs, OB stars, or evolved stars undergoing mass loss. Dust surrounding evolved stars is less frequently detected than from protostars. Only a handful of such objects up to a distance of $5 \mathrm{kpc}$ are detected at $870 \mu \mathrm{m}$ with LABOCA within the sensitivity limit (on average $\sigma \sim$ $70 \mathrm{mJy} / \mathrm{beam}$ ) of ATLASGAL (Ladjal et al. 2010). Since the majority of the evolved stars are expected to emit weakly in the submillimeter, the ATLASGAL sources are expected to be dominated by young, star-forming objects. As a consequence, their association with mid-infrared sources can generally be used as a proxy to trace ongoing star formation.

MSX surveyed the Galactic plane at mid-infrared wavelengths with an angular resolution $(18 . " 3$ at $8-21 \mu \mathrm{m})$ similar to that of the ATLASGAL survey. With a higher spatial
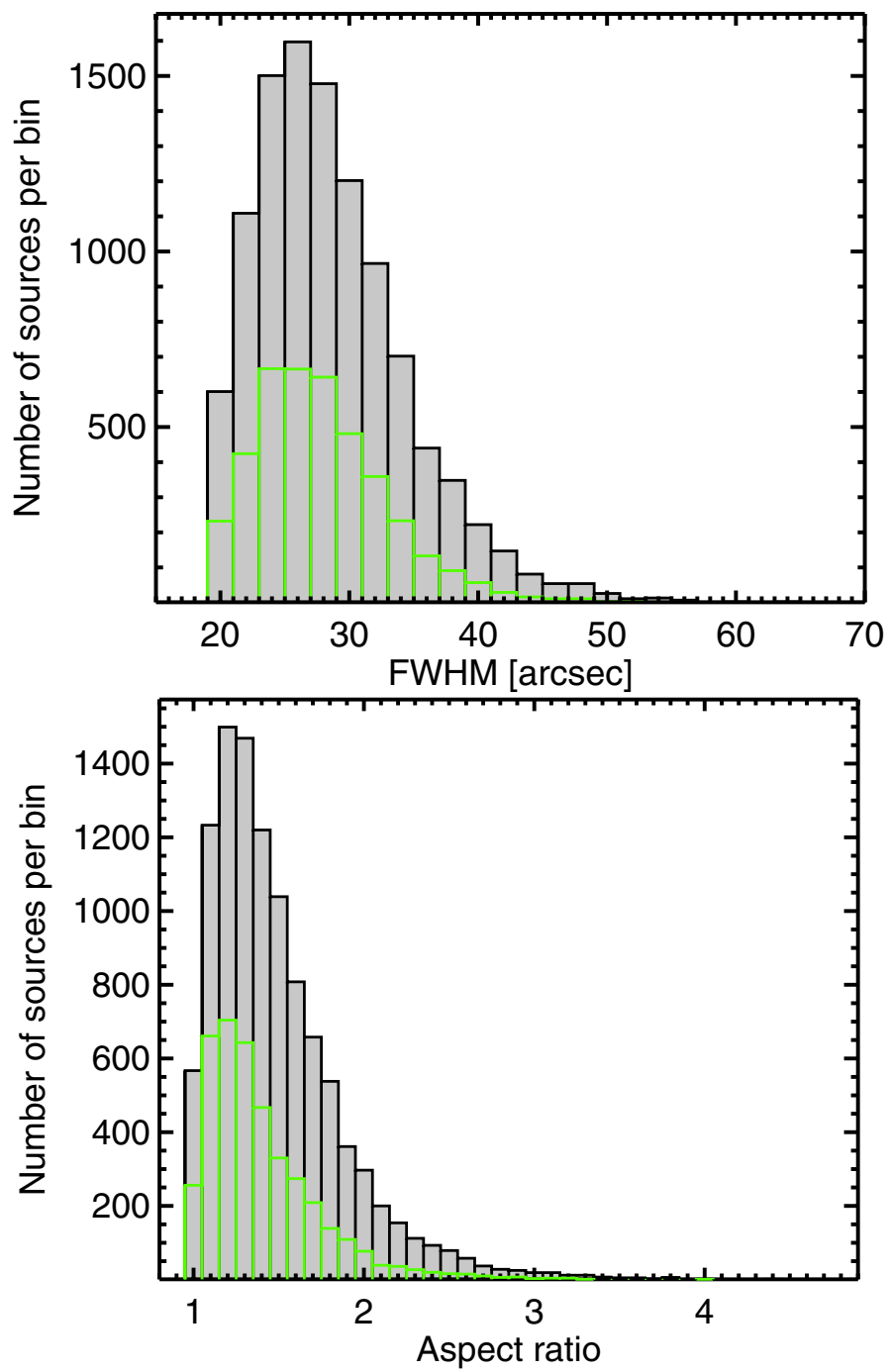

Fig. 9. Top: distribution of the beam convolved FWHM sizes of the detected sources. The green line shows the ATLASGAL sources with midinfrared associations (see Sect. 5 for details). Bottom: same as top panel for the aspect ratio of the detected sources.

resolution and sensitivity, surveys with the Spitzer and WISE space telescopes uncover a larger population of mid-infrared sources in the Galactic plane. The multicolor Spitzer GLIMPSE and MIPSGAL surveys probe fainter, thus even younger or lower mass protostars, and they represent the highest spatial resolution and brightness sensitivity at mid-infrared wavelengths to date. However, due to source confusion it is not straightforward to unambiguously associate Spitzer sources with dust peaks, and the line-of-sight contamination from chance alignments with field stars is high. MIPSGAL at $24 \mu \mathrm{m}$ has only a factor of 2 higher sensitivity and an angular resolution of $6^{\prime \prime}$ (Carey et al. 2009) compared to WISE at $22 \mu \mathrm{m}$ with $12^{\prime \prime}$ resolution. Since here we only study the statistical properties of the mid-infrared associations with ATLASGAL sources, the best combination of surveys are the MSX and WISE datasets with available point source catalogs. They cover a continuous brightness sensitivity from the brightest sources in the Galactic plane to weaker, deeply embedded young protostars and have a comparable angular resolution to ATLASGAL at $870 \mu \mathrm{m}$.

Contreras et al. (2013) used data only from MSX at $21.3 \mu \mathrm{m}$ to give a crude estimate of the proportion of star-forming clumps of ATLASGAL. We base the characterization of the mid-infrared 
T. Csengeri et al.: ATLASGAL - a catalog of dust condensations in the Galactic plane

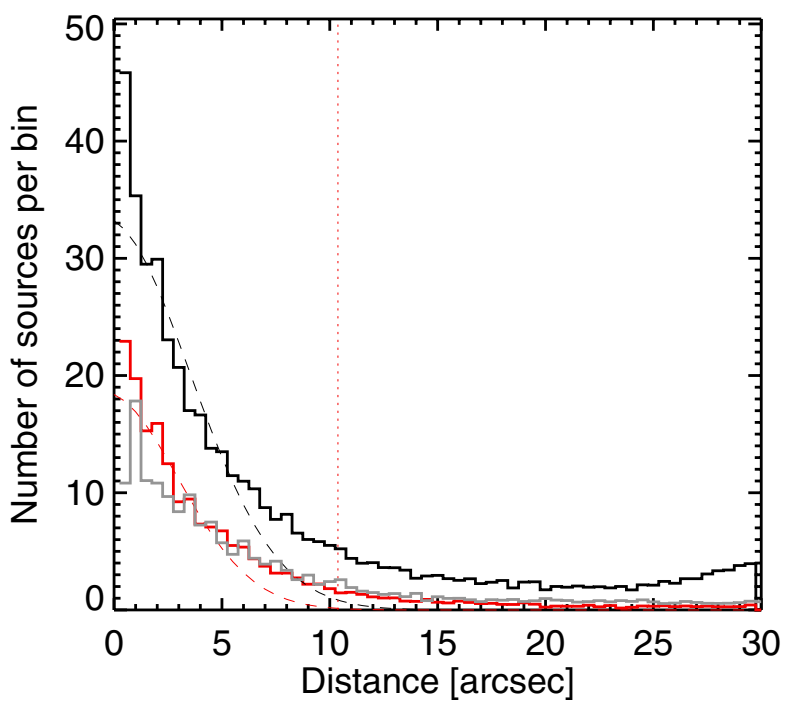

Fig. 10. Distribution of the normalized distance between the dust continuum peaks and the mid-infrared sources within $30^{\prime \prime}$. Black line shows all sources from the WISE catalog, while the red line corresponds to the distribution of only the red sources (see text for details). As a comparison the same distribution for all MSX sources is shown in gray. Dashed lines show the Gaussian fits to these distributions, the maximum angular distance between mid-infrared sources, and ATLASGAL sources was determined as $3 \sigma$ from this and is shown with a dashed line.

content of dust clumps using the $21.3 \mu \mathrm{m}$ filters on MSX (E-band) and the $22 \mu \mathrm{m}$ filter on WISE (band 4). The former provides a sensitivity down to 2-6 Jy (Egan et al. 1999), while the latter has a $5 \sigma$ point source sensitivity in unconfused regions of 6 mJy (Wright et al. 2010). MSX suffers from saturation only for the brightest, a few hundred Jy bright sources, and although the WISE $22 \mu \mathrm{m}$ band starts to saturate at $\sim 10 \mathrm{Jy}$, reliable photometry can be extracted up to $330 \mathrm{Jy}$ by fitting the wings of the PSF (Cutri et al. 2012). Therefore the two catalogs have a substantial overlap in sensitivity (see also Sect. 5.3), and combining these gives a continuous $21-22 \mu \mathrm{m}$ sensitivity from $6 \mathrm{mJy}$ to several hundred Jy, covering five orders of magnitude.

In Sect. 5.1 we compare source counts using the $21 \mu \mathrm{m}$, as well as the more sensitive $8 \mu \mathrm{m}$ ( $A$-Band), information from MSX. This dataset is relevant for the brightest sources and is directly comparable to the mid-infrared analysis of Contreras et al. (2013). In Sect. 5.2 we use the more sensitive WISE point source catalog to complement this sample and analyze the color properties of the ATLASGAL-mid-infrared associations. We merge these two samples to derive the final number of star-forming ATLASGAL sources in Sect. 5.3 and study their characteristic colors in Sect. 5.4.

\subsection{Association with MSX}

We have searched for MSX counterparts of ATLASGAL sources within a $60^{\prime \prime}$ search radius using the MSX Point Source Catalog (PSC) v2.3 (Egan et al. 2003). In the next step we determined a more appropriate matching radius by fitting a Gaussian to the normalized distribution of the angular separation between the MSX sources and ATLASGAL peak positions and then take $3 \sigma$ as a match radius corresponding to $\sim 13^{\prime \prime}$ (Fig. 10).

We find a total number of 1669 sources to have a corresponding MSX source within this angular separation and with detectable flux at $21.3 \mu \mathrm{m}$. This corresponds to $16 \%$ of the total ATLASGAL sources (Table 2) and is a more conservative
Table 2. Summary of cross-matches of dust condensations identified in the ATLASGAL survey.

\begin{tabular}{rrr}
\hline \hline Catalog & Matched sources & Fraction [\%] \\
\hline MSX-ATLASGAL & 1669 & 16 \\
WISE-ATLASGAL & 3151 & 30 \\
MSX-WISE-ATLASGAL & 3483 & 33 \\
\hline
\end{tabular}

estimate than the $34.8 \%$ found by Contreras et al. (2013). Using a similar 30" search radius to Contreras et al. (2013), we find the same fraction of the sources with an MSX counterpart.

We compare the color properties of these $16 \%$ matches with the color selection criteria used in the literature to search for embedded massive objects. We find that $97 \%$ of the sources fulfill the minimum criteria of $F_{E}>F_{D}$, which are the flux measurements at $14.65 \mu \mathrm{m}$ ( $D$-band) and $21.3 \mu \mathrm{m}$ ( $E$-Band), and this corresponds to a rising SED towards longer wavelengths. Schuller et al. (2006) use a selection criteria of $F_{E} / F_{D} \geq 2$, and from our matches we find that 1165 fulfill this, which is $77 \%$ of the sources with reliable flux measurements in both of these bands. Lumsden et al. (2002) adopts a less conservative requirement by using $F_{E} \geq 2 \times F_{A}$, and we find that $86 \%$ of our matches fulfill this, where $F_{A}$ corresponds to the flux in the $A$ band of MSX $(8 \mu \mathrm{m})$. We note that extending the match radius to $30^{\prime \prime}$ leads to a lower fraction of sources fulfilling these color properties, suggesting that the larger match radius would contain more sources with chance line-of-sight alignment or merging of nearby sources with a different evolutionary status. Our position-restricted estimate of the mid-infrared content of ATLASGAL sources therefore corresponds to mostly embedded objects, while it is likely that the Contreras et al. (2013) matches include more significant contamination of chance alignment sources.

Since MSX has the highest sensitivity in the $8 \mu \mathrm{m}$ band (0.1-0.2 Jy, Egan et al. 2003), we derive source counts of ATLASGAL matches in this band, while following the same method as described above. Similar to above, we derive a $13^{\prime \prime}$ distance limit here and find 2238 sources matched to dust condensations. This number represents $21 \%$ of the ATLASGAL sources. Out of these, 1647 fulfill our selection criteria of the $21 \mu \mathrm{m}$ matches, and the rest are new associations. From these sources (only detected at $8 \mu \mathrm{m}$ and not at $21 \mu \mathrm{m}$ ), we find 130 sources that are only detected at the shortest wavelengths (i.e., not detected in the $C$ and $D$ bands), suggesting that some of these new associations may be contaminated by evolved stars or PDR emission features. Therefore, despite the higher sensitivity, we do not significantly increase the fraction of star-forming clumps using the $8 \mu \mathrm{m}$ of MSX instead of the $21 \mu \mathrm{m}$ band.

We note that between $8 \mu \mathrm{m}$ and $22 \mu \mathrm{m}$, not only does the heated dust from the inner envelope emit, but there is diffuse emission from polycyclic aromatic hydrocarbon (PAHs) in the surrounding nebulosity. For the most complex region in the Galactic center, Schuller et al. (2006) estimates $10 \%$ of the sources to be actually diffuse PDR emission rather than protostellar sources.

\subsection{Association with WISE}

Since WISE is more sensitive than MSX in all bands (by a factor of 500 at $21 \mu \mathrm{m}$ ), there is a higher probability of chance alignment between WISE and ATLASGAL sources. These can be field stars, low-mass YSOs and brown dwarfs from the 
foreground, and evolved stars. In the following we use the WISE point source catalog (Wright et al. 2010), and with a 30" search radius we find a $96 \%$ match between ATLASGAL and WISE sources. We investigated the color properties of these first associations based only on positional coincidence in order to get a first hint of their nature. In fact, as expected, we found that such associations are largely contaminated by chance alignment with field stars.

It is necessary therefore to determine a more reliable estimate of the number of embedded mid-infrared sources associated with ATLASGAL clumps by eliminating chance alignments as far as possible. For this we first limited the search radius to find the best potential matches (Fig. 10) using a similar strategy to the one discussed above for MSX (see Sect. 5.1). However, given the large number of WISE matches, we studied the distribution of the angular offset between mid-infrared and dust peaks using only embedded sources with characteristic red colors $\left(F_{4.5 \mu m} / F_{3.6 \mu m}>0.75\right.$ and $F_{4.5 \mu \mathrm{m}} / F_{12 \mu m}<1^{7}$, Lumsden, priv. comm.). We arrived at a similar search radius of $10^{\prime \prime}$ to the one for MSX. For the eventual matching between the ATLASGAL and WISE positions, we did not use any color requirements, but the above derived limiting angular offset. We then put a further constraint on the matched sources by requiring a $S / N>10$ for the $22 \mu \mathrm{m}$ band WISE measurements in order to ensure their nature as embedded sources instead of a chance alignment with a field star or evolved stars, which are not detected at $22 \mu \mathrm{m}$ (see, e.g., Schuller et al. 2006). We discuss in more detail the justification and the color properties of these matches in Sect. 5.4.

With these selection criteria we find 3151 ATLASGAL sources to have a WISE association. This corresponds to $30 \%$ of all the ATLASGAL sources.

From these matches we find that only a negligible fraction have $\mathrm{S} / \mathrm{N}$ lower than 2 or a bad quality flag ${ }^{8}$ at $12 \mu \mathrm{m}$ (band 3 ). Therefore the majority $(89 \%)$ of our matches are detected at least in two WISE bands. Interestingly, we find that $97 \%$ of these matches have a measured flux at $4.5 \mu \mathrm{m}$ (band 2) with a $\mathrm{S} / \mathrm{N}$ greater than 2 (this value drops to $93 \%$ when restricting to $S / N>10)$. In the $4.5 \mu \mathrm{m}$ and the $12 \mu \mathrm{m}$ bands, we find that $86 \%$ of the sources are detected with $S / N>2$. At the shortest wavelengths $(3.6 \mu \mathrm{m}$, band 1) $89 \%$ of the matched sources have fluxes above $2 S / N$. Altogether $82 \%$ of our matches (2559 sources) have fluxes measured in all four bands above $S / N>2$.

From these 2559 sources, 2481 (97\%) fulfill the [3.6] > [4.5] $>[12]>[24]$ criteria corresponding to rising SEDs in the mid-infrared wavelength range. We find that $96 \%$ of them also fulfill the red object selection criteria used above $\left(F_{4.5 \mu \mathrm{m}} / F_{3.6 \mu \mathrm{m}}>0.75\right.$ and $\left.F_{4.5 \mu \mathrm{m}} / F_{12 \mu \mathrm{m}}<1\right)$, while $99 \%$ of them fulfill the $F_{22} \mu \mathrm{m}>2 \times F_{12 \mu \mathrm{m}}$ criteria, which is similar to that of Lumsden et al. (2002). The majority of these associations are therefore reddened, most likely embedded star-forming objects. We study the color properties of this sample in more detail in Sect. 5.4.

In the following we refer to ATLASGAL sources with associated mid-infrared emission as star-forming clumps, and to the rest of the sources as quiescent. They represent a lower limit to the true proportion of star-forming sources since there is likely to be a number of clumps associated with embedded sources that fall below the $10 \sigma$ detection threshold we used for the WISE catalog.

\footnotetext{
7 This corresponds to [3.6] $-[4.5]>0.24$ and [4.5] $-[12]<0.0$.

8 Objects flagged as "D", "H", "O", or "P" are likely to be spurious sources according to the WISE catalog description.
}

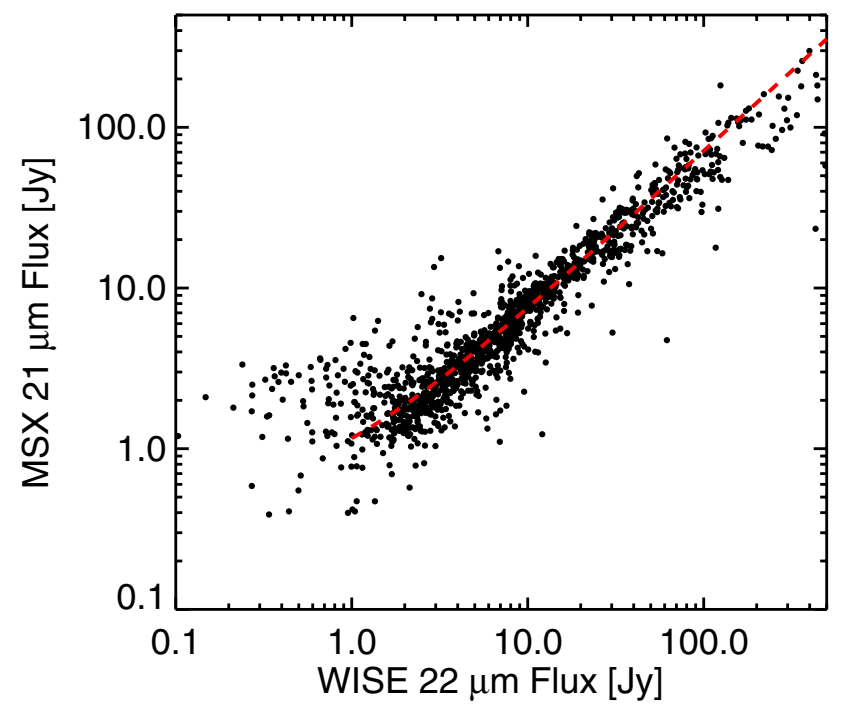

Fig. 11. Comparison of the WISE $22 \mu \mathrm{m}$ and MSX $21 \mu \mathrm{m}$ fluxes for the sources that are found in both catalogs. Red dashed line shows a robust linear fit with a slope of $0.71 \pm 0.01$.

\subsection{Correlation between $21 \mu \mathrm{m}$ and $870 \mu \mathrm{m}$ fluxes of star-forming ATLASGAL sources}

Comparing the 21-22 $\mu \mathrm{m}$ flux densities between the MSX and WISE sources, we find that 1308 sources are found in both catalogs. To check our independently matched ATLASGAL sources with MSX and WISE, we show in Fig. 11 the comparison of the $21 \mu \mathrm{m}$ flux density from the two catalogs 9 . These independently derived flux densities from both surveys show good correlation (with a Pearson correlation coefficient of 0.88 for sources between 10-80 Jy fluxes); however, it is also clear that below $\sim 10 \mathrm{Jy}$, the flux measurements of MSX show increased scatter. This is fully consistent with the varying noise level in the MSX data at different positions in the Galactic plane, leading to less reliable measurements at the lower flux limit. For the brightest sources, the WISE photometry seems to give systematically higher fluxes.

Taking this overlap between the two catalogs into account, we arrive at a total number of 3483 associations at $21-22 \mu \mathrm{m}$ with ATLASGAL sources. This is $33 \%$ of the total number of dust condensations from the ATLASGAL catalog. This result compares well with Contreras et al. (2013), who derive an upper limit on the fraction of star-forming clumps of $\sim 50 \%$ using a less robust test for mid-infrared emission with MSX. Here our matching criteria is more conservative to associate embedded sources with the ATLASGAL clumps (see also Sect. 5.4), resulting in a more robust sample. Therefore our derived fraction is a strict lower limit of the fraction of star-forming dust clumps in the inner Galaxy.

Figure 12 shows the measured $22 \mu \mathrm{m}$ flux densities from WISE and MSX versus the $870 \mu \mathrm{m}$ flux density from ATLASGAL. The mid-infrared flux density seems to increase with increasing submillimeter flux density; in particular, the brightest dust clumps exhibit brighter $22 \mu \mathrm{m}$ fluxes. We determined the Pearson coefficient to check for a correlation between these two parameters using the WISE sources with fluxes between $10-300 \mathrm{Jy}$, where the photometry is the most reliable. We obtain a correlation coefficient of 0.29 with a significance value

\footnotetext{
9 We convert the magnitudes given in the WISE catalog using the zerolevel fluxes from Wright et al. (2010), a color correction factor of 1.0, and a correction factor of 0.9 from Cutri et al. (2012).
} 
T. Csengeri et al.: ATLASGAL - a catalog of dust condensations in the Galactic plane

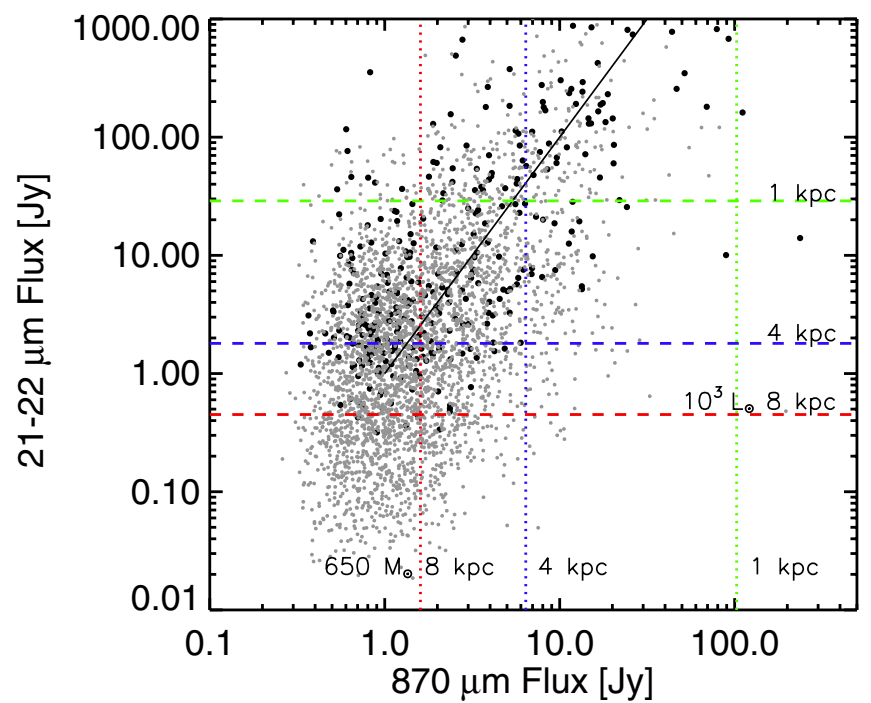

Fig. 12. Integrated flux density from ATLASGAL versus the flux density at $22 \mu \mathrm{m}$. Gray dots show the WISE, and black dots show the flux measurement from MSX associations. For sources found in both the WISE and MSX catalogs, only the WISE flux is shown. The brightest sources tend to have higher $22 \mu \mathrm{m}$ flux (see text for more details). Dashed lines show the flux limit at $22 \mu \mathrm{m}$ corresponding to a B3 protostar at 1,4 , and $8 \mathrm{kpc}$. The mass limit for high-mass star-forming clumps at the same distances is shown with dotted lines. The black solid line shows a relation of $S_{22 \mu \mathrm{m}} \sim S_{870 \mu \mathrm{m}}^{2}$.

of $<0.001$, which suggests a weak correlation between these parameters. As a comparison we plot a relation of $S_{22 \mu \mathrm{m}} \sim S_{870 \mu \mathrm{m}}^{2}$ to highlight the trend seen in the data. This may reflect that the more massive and luminous YSOs form within more massive dust clumps, producing the distinct tail of the distribution; however, distance and temperature effects may also play a role.

We also show in Fig. 12 the $22 \mu \mathrm{m}$ flux density limit corresponding to a ZAMS B3 type star with a bolometric luminosity of $10^{3} L_{\odot}$ at 1,4 , and $8 \mathrm{kpc}$ distance based on the color properties defined by Wood \& Churchwell (1989) and adopted here following Motte et al. (2007). We also show the mass limit required to host MDCs, precursors to high-mass stars, corresponding to $650 M_{\odot}$ (see Sect. 3.5) at these distances. We adopt here a dust temperature of $18 \mathrm{~K}$, because on larger scales the dust temperature is dominated by the interstellar radiation field at approximately this value (Bernard et al. 2010). Similar values have been used by Motte et al. (2007), who adopt $T=20 \mathrm{~K}$ for MDCs and Wienen et al. (2012) as well, who determine a gas kinetic temperature of $17 \mathrm{~K}$ for a sample of ATLASGAL clumps.

For 1,4 , and $8 \mathrm{kpc}$, this mass limit translates to a submillimeter flux density limit of 102.6, 6.4, and 1.6 Jy, respectively, in order to potentially form high-mass stars. To eventually determine the fraction of massive clumps, distance information is essential (Wienen et al. 2012).

Figure 13 shows the histogram of the ratio of the $22 \mu \mathrm{m}$ and $870 \mu \mathrm{m}$ fluxes. We find a broad range for this flux ratio with a peak around one. We also plot the ATLASGAL-MMB, the ATLASGAL-CORNISH, and the ATLASGAL-RMS associations from Urquhart et al. (2013a and b), respectively. The MMB sources tend to show a lower flux ratio than the CORNISH and RMS samples. We note, however, that the CORNISH sample can be considered as a subsample of the RMS sources because they consist of MYSOs and H II regions. The peak of the distribution for all star-forming ATLASGAL sources is shifted towards lower flux ratios than for the MMB and more evolved

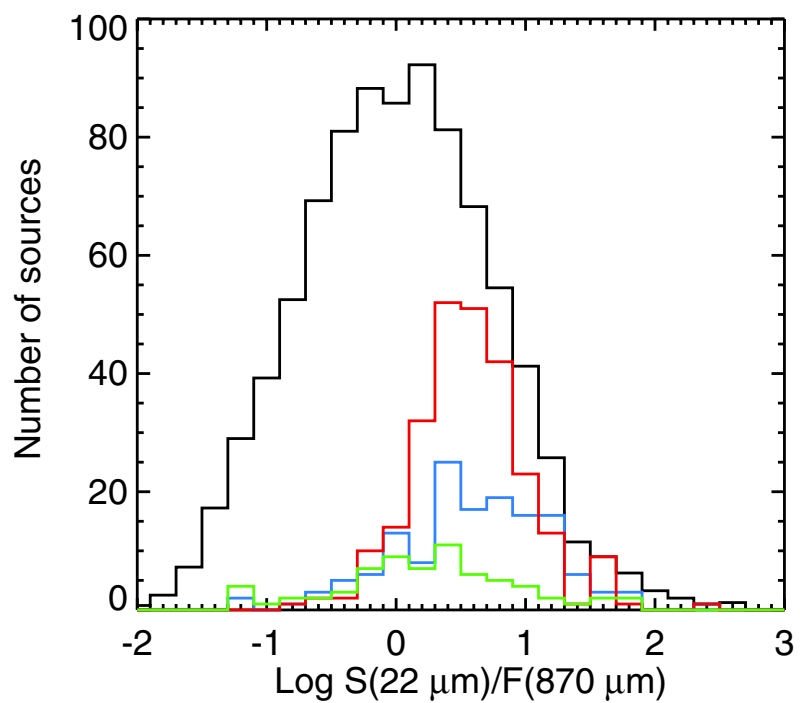

Fig. 13. Histogram of the ratio of $22 \mu \mathrm{m}$ and $870 \mu \mathrm{m}$ peak fluxes. The black line shows the whole sample (scaled down by a factor of 4 ), red line shows the RMS associations, blue corresponds to the CORNISH sources, while green indicates the MMB sample.

samples, suggesting an evolutionary trend with lower flux ratios corresponding to colder sources.

\subsection{Infrared colors of ATLASGAL sources}

We use the WISE matched ATLASGAL sources which have measured fluxes above $2 \mathrm{~S} / \mathrm{N}$ in all bands (2559 objects, $25 \%$ of the identified ATLASGAL sources) to study their characteristic colors. To demonstrate the results of these matches we show a three-color composite image in Fig. 14 with the starforming ATLASGAL sources indicated together with all the WISE sources found within a $30^{\prime \prime}$ search radius. We also use the list of ATLASGAL matched MMB associations (Urquhart et al. 2013a), CORNISH sources (Urquhart et al. 2013b) and RMS sources (Urquhart et al., in prep.) to illustrate the location of these sources with a known evolutionary stage on these plots. In general there are several WISE sources found within the $30^{\prime \prime}$ search radius, and a substantial fraction of them are field stars appearing in blue in Fig. 14. The requirement of emission at $22 \mu \mathrm{m}$, however, eliminates a major number of these sources.

As shown in Fig. 15 (as well as Figs. B.1-B.4), the majority of these matches separate well from field stars in the colorcolor space, suggesting that the contamination of chance alignments is low. Clearly, the mid-infrared sources associated with ATLASGAL clumps are deeply embedded, showing characteristic reddened colors. Their average colors are summarized in Table 3 . The outlying points on the diagram probably correspond to mismatched sources, where our method erroneously associated a nearby field star to the ATLASGAL source. This may happen in complex regions affected by saturation, where the $22 \mu \mathrm{m}$ flux measurement in the WISE catalog is flagged, but there is a nearby red source that can be a chance alignment.

Comparing the positions of embedded H II regions and of RMS and MMB sources in Fig. 15, we see that especially the first two occupy a well-defined region with colors between $1.5<[3.4]-[4.6]<6$ and $2<[12]-[22]<8$. These sources correspond to more evolved stages of high-mass star formation, H II regions, and MYSOs. The H II regions seem to occupy a well-determined region of the plot, while the RMS sources show more dispersion, very likely because this sample is a 


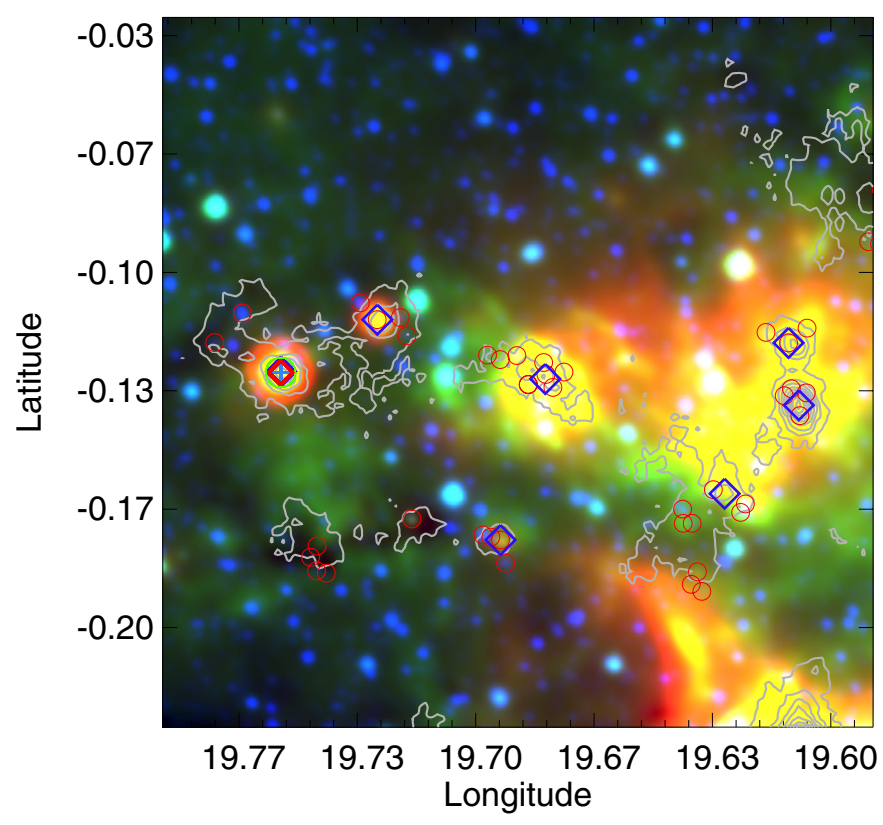

Fig. 14. Example showing the distribution of ATLASGAL and WISE point sources on a color composite image from WISE (blue: $3.4 \mu \mathrm{m}$; green: $12 \mu \mathrm{m}$; red: $22 \mu \mathrm{m}$ ) with the $870 \mu \mathrm{m}$ contours overlaid from ATLASGAL. Red circles show all the WISE sources within $30^{\prime \prime}$ of the ATLASGAL sources, and dark blue diamonds indicate the associated ATLASGAL and WISE positions likely hosting embedded sources. Light-blue crosses mark the position of CORNISH sources (Urquhart et al. 2013b), while green circles show the positions of all MMB sources (Urquhart et al. 2013a). Thick red circles show the RMS sources (Urquhart et al., in prep.).

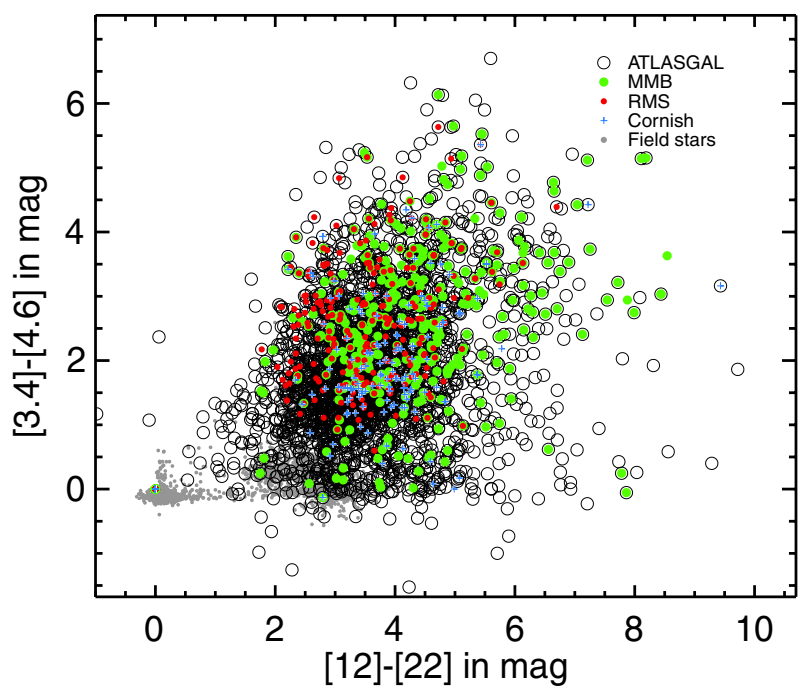

Fig. 15. Color-color plots of WISE matched ATLASGAL sources with measurable WISE fluxes in all bands. Field stars from a randomly chosen $\sim 0.5^{\circ} \times 0.5^{\circ}$ area on the sky around the central coordinates of $\ell=280^{\circ}, b=15^{\circ}$ are shown in gray.

combination of MYSOs and H II regions. The largest scatter is seen for the MMB sources, which includes many of the more extreme reddened objects than the two other samples. This is consistent with the general view of the occurrence of Class II methanol masers, which predominantly trace high-mass star formation and are also frequently found around UC-H II regions (e.g., Urquhart et al. 2013a).
Table 3. Mean and standard deviation of WISE colors of dust condensations.

\begin{tabular}{rrrrr}
\hline \hline Sample & {$[3.6]-[4.6]$} & {$[4.6]-[12]$} & {$[12]-[22]$} & {$[4.6]-[22]$} \\
\hline AG-WISE & $1.76 \pm 1.02$ & $3.48 \pm 1.56$ & $3.45 \pm 0.93$ & $6.93 \pm 1.74$ \\
CORNISH & $1.56 \pm 1.28$ & $3.34 \pm 2.35$ & $2.89 \pm 1.94$ & $6.23 \pm 3.94$ \\
RMS & $0.86 \pm 1.36$ & $1.04 \pm 1.62$ & $1.18 \pm 1.77$ & $2.21 \pm 3.30$ \\
MMB & $1.40 \pm 1.57$ & $1.51 \pm 1.86$ & $2.31 \pm 2.37$ & $3.82 \pm 3.78$ \\
\hline
\end{tabular}

A large number of the unclassified ATLASGAL sources are spread in the color space, similarly to the MMB sources suggesting that they may host the early stage of intermediate- and high-mass star formation. There are several sources with moderately reddened colors, and they are likely to be low- to intermediate-mass nearby star-forming cores. Using this plot we identify a number of highly reddened extreme sources that have not been revealed by the other surveys. These may be of potential interest to study in greater detail.

\section{Galactic distribution of star-forming and quiescent ATLASGAL sources}

\subsection{Galactic longitude}

The distribution of quiescent and star-forming ATLASGAL sources as a function of Galactic longitude is shown in Fig. 16. With over 10000 sources we are able to trace various aspects of the Galactic structure. The strongest peak corresponds to the Galactic center region, with the highest number density of ATLASGAL sources. We see additional overdensities toward the direction of the Scutum arm, the Norma arm, and Sagittarius arm. In addition, there are several known star-forming complexes appearing as statistically significant $(>7 \sigma)$ peaks. The G305 complex stands out from the average source density, but we find other peaks that indicate massive dust complexes, such as those around $\ell=327^{\circ}, \ell=333^{\circ}$ towards the Norma-tangent, and $\ell=337^{\circ}$, which is in the direction of the $3-\mathrm{kpc}$ arm. These are spatially very extended complexes rich in embedded sources. The $\ell=333^{\circ}$ complex contains sources mostly at about $3.5 \mathrm{kpc}$ (Simpson et al. 2012) in the Crux arm, between the Sagittarius and Norma arms along the line of sight (Bronfman et al. 2000). The source counts in Fig. 16 are likely to be lower beyond $\ell=327^{\circ}$, because there is only one arm on the line of sight with the Sagittarius arm being quite off of the plane. Likewise, the $\ell=337^{\circ}$ region is in the tangent region of the $3-\mathrm{kpc}$ arm, but there is strong molecular emission both from the Norma and the Crux spiral arms. Therefore the peaks in Fig. 16 are related to the superposition of spiral arms on the line of sight. We also find a narrow peak toward $\ell=345^{\circ}$. In this region there are several clouds spread over relatively high Galactic latitudes between $b=-0.9^{\circ}$ to $b=1.3^{\circ}$. These features are all found within a very narrow Galactic longitude range of $345.2^{\circ}<\ell<345.5^{\circ}$, and as a consequence they appear as a very narrow peak in Fig. 16. This complex has been studied in more detail in López et al. (2011).

The relatively nearby complexes of NGC 6334 and NGC 6357 (Russeil et al. 2010) also appear. The peaks at positive Galactic longitudes at $\ell=10-12^{\circ}$ correspond to the complexes associated with W31 and W33, and there is a clear excess of sources around $\ell=15-17^{\circ}$ dominated by the known starforming regions M16 and M17. There are other peaks associated with mini starburst regions like W43 and W51. Interestingly, the active star-forming region, W49, does not appear as a prominent 
T. Csengeri et al.: ATLASGAL - a catalog of dust condensations in the Galactic plane

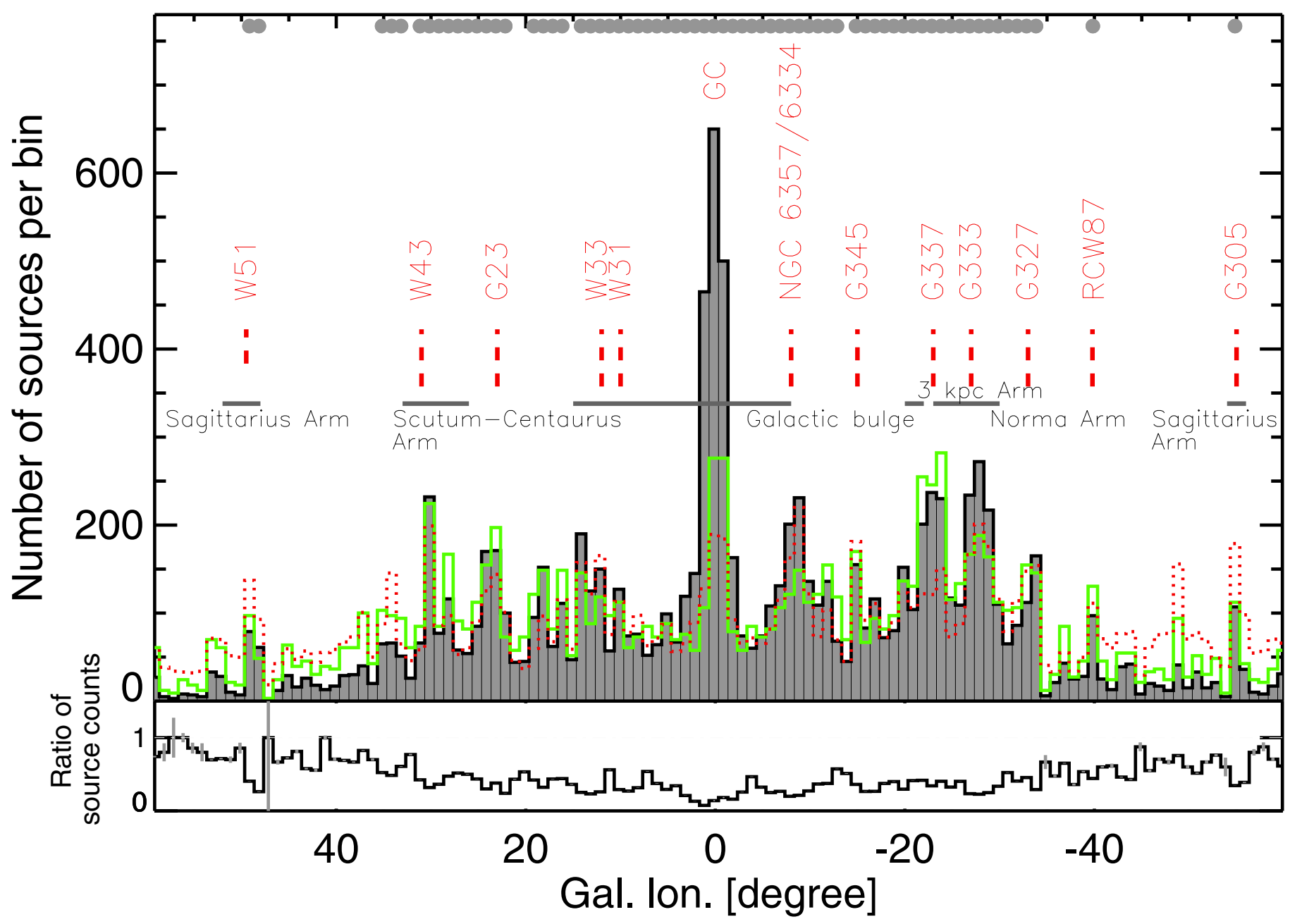

Fig. 16. Top: distribution of the ATLASGAL sources in Galactic longitude binned in $1^{\circ}$. Gray lines indicate the spiral arms and the dust complexes are labeled in red. Gray dots in the upper line of the panel show the bins with $>7 \sigma$ peaks. The green line shows the distribution of star-forming ATLASGAL sources and have been scaled to the dust source counts. Red dotted line shows the WISE selected red sources according to the color criteria (see text for details). Bottom: the distribution of the ratio of star-forming to quiescent ATLASGAL sources is shown in black. Error bars in gray indicate the propagated Poisson error from the two distributions.

peak. This can be explained by its greater distance of $12 \mathrm{kpc}$ (Gwinn et al. 1992) than for the other regions, such as W43 and W51, which are located at 6 and $5.4 \mathrm{kpc}$, respectively (Nguyen Luong et al. 2011; Sato et al. 2010). As a consequence, toward W49 the physical resolution is not sufficient to resolve individual clumps, and only a few massive cloud fragments are identified. Alternatively, another difference could arise from the different stage of evolution between molecular complexes.

The Galactic structure traced by the population of compact sources shown in Fig. 16 is in general very similar to what is reported by Beuther et al. (2012), who use the same data but a different source identification method. In Fig. 16 we also show the normalized Galactic distribution of the star-forming ATLASGAL sources. These correlate well with the positions of the peaks associated with molecular complexes as discussed above. The fraction of star-forming versus quiescent sources is lower between $-35^{\circ}<\ell<35^{\circ}$, with the lowest ratio found towards the Galactic center region. This ratio suggests a lower star formation rate (SFR) toward the Galactic center region, although this strikingly low ratio of star-forming versus quiescent sources can also be partially due to much higher extinction (see also Beuther et al. 2012). Using various datasets, other authors arrive at the same conclusion, i.e., that the Galactic center region has an exceptionally low SFR (Immer et al. 2012; Urquhart et al. 2013a; Longmore et al. 2013). The mechanism responsible for the lack of star formation in this region has been investigated by Kruijssen et al. (2014), however no single physical mechanism (turbulence, tidal forces, feedback effects, cosmic ray heating, etc.) was identified as sufficient for suppressing the SFR to such low levels. In contrast to the Galactic center region, the $|\ell|>35^{\circ}$ range, the SFR seems to be rather uniform, the fraction of ATLASGAL sources exhibiting star formation activity is $67 \pm 17 \%$ on average.

As a comparison, we selected sources from the WISE catalog covering the same area as ATLASGAL and put a color cut as a selection criterion of $\left(F_{4.5 \mu \mathrm{m}} / F_{3.6 \mu \mathrm{m}}>0.75\right.$ and $F_{4.5 \mu \mathrm{m}} / F_{12 \mu \mathrm{m}}<1$ ) (Lumsden, priv. comm.) to select red objects. Here we find that the embedded sources have a comparable distribution to the dust peaks. Compared to the Robitaille et al. (2008) GLIMPSE YSOs used in Beuther et al. (2012), we find that many of the YSO peaks coincide with the dust peaks, most likely because our sample of red embedded objects is more complete than in the other studies. In the $|\ell|>35^{\circ}$ range, however, there is an increase in the number of embedded red objects 


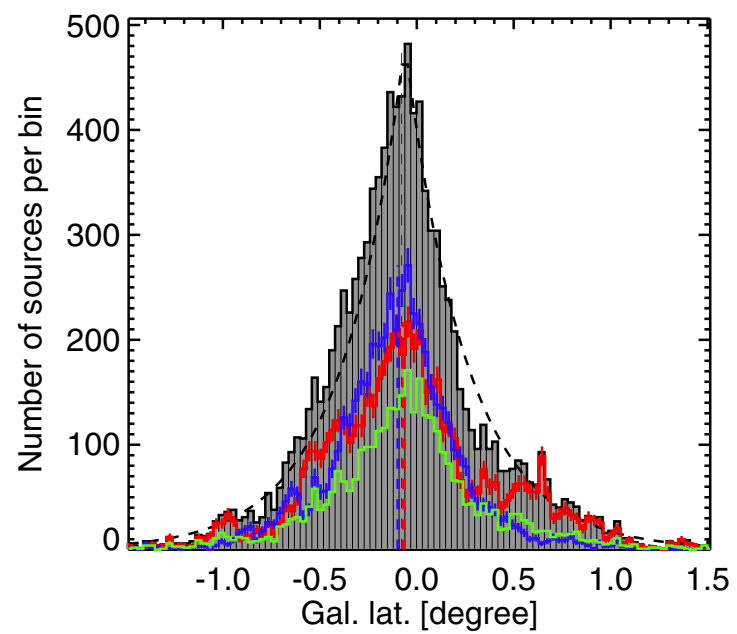

Fig. 17. Distribution of the detected sources binned in Galactic latitude. The gray histogram corresponds to all detected sources, the blue line shows those lying in the Ist Galactic quadrant, and red line shows sources from the IVth quadrant. The dashed line shows the function $400 \mathrm{e}^{-|b| / 0.32^{\circ}}$ suggesting a $0.32^{\circ}$ scale height for the ATLASGAL sources. Dashed colored lines correspond to the peak position of the Ist and IVth quadrant source distributions. The green line shows the normalized distribution of star-forming ATLASGAL to the total number of sources.

with respect to the dust source counts, which is very likely due to higher contamination of YSOs, as well as of evolved stars. In this sample this excess is clearly seen in the $|\ell|>35^{\circ}$ range, where more nearby sections of the Galactic arms fall, hence the infrared source sample, starts to be dominated by nearby lowmass embedded objects.

\subsection{Galactic latitude}

Despite a similar number of sources being found below and above the Galactic plane, the Galactic latitude distribution of ATLASGAL sources is asymmetric and shows a characteristic shift towards negative latitudes (Fig. 17). We determine an offset of $-0.076^{\circ} \pm 0.006^{\circ}$ to the peak of the distribution, which is very similar to the value of $-0.07^{\circ}$ determined by Beuther et al. (2012). Other studies using similar datasets have also reported this shift (e.g., Schuller et al. 2009; Rosolowsky et al. 2010), such as studies using various other tracers of (massive) star formation, i.e. UC-H II regions, (Bronfman et al. 2000), GLIMPSE YSOs (Robitaille et al. 2008), and molecular gas (Cohen \& Thaddeus 1977; Bronfman et al. 1988). This shift is greater when only using IVth quadrant sources. However, this is not seen for samples of ATLASGAL sources associated with UC-H II regions and MMB sources. It is therefore likely that in ATLASGAL a large number of local clumps are seen tracing the Sun's position off the Galactic plane.

We estimate the Galactic scale height for dust condensations using an exponential function and find that a value of $0.32^{\circ}$ gives a good fit to the data. This means that the majority of dust sources are confined to a very narrow region around the Galactic midplane. As a comparison, the scale height of OB stars from IRAS is $0.6^{\circ}$ derived by Wood \& Churchwell (1989), which is similar to the $0.8^{\circ}$ using MSX data to select OB type stars by Lumsden et al. (2002). Our estimate for the embedded stages is therefore much lower than when based only on mid-infrared diagnostics. This can be partly explained with contamination of the previous samples with evolved stars, which has been claimed for

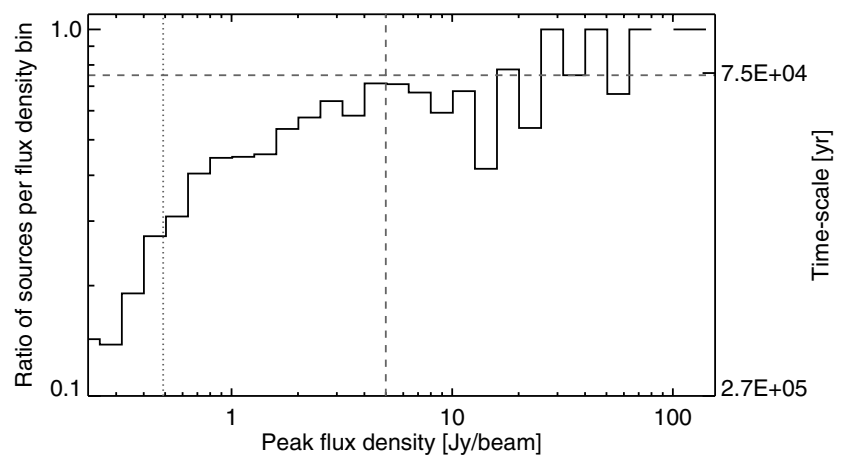

Fig. 18. Ratio of star-forming versus all ATLASGAL sources as a function of peak flux. The $75 \%$ is indicated by a dashed line. This fraction is fulfilled above a $5 \mathrm{Jy}$ beam-averaged flux density. The dotted line shows the $7 \sigma$ completeness level of the catalog.

the Wood \& Churchwell (1989) sample by Becker et al. (1994), who estimate $0.4^{\circ}$ scale height using UC-H II regions. Recent studies suggest a lower scale height closer to the value suggested from ATLASGAL, and Walsh et al. (2011) estimates $0.4^{\circ}$ using $\mathrm{H}_{2} \mathrm{O}$ masers, similarly to Urquhart et al. (2011). Our estimate of a $0.32^{\circ}$ scale-height corresponds to $47 \mathrm{pc}$ at $8.4 \mathrm{kpc}$ (Reid et al. 2009), which is the same as derived by Beuther et al. (2012).

We also report here inhomogeneities in the distribution of IVth and Ist quadrant sources. The most prominent is an excess in sources at negative latitudes around $\sim-1.0^{\circ}$. This bump seems to be associated to sources from the IVth quadrant.

As a comparison, the normalized distribution the starforming ATLASGAL sources is shown in Fig. 17. The starforming ATLASGAL sources are well fit with the same scale height as for the total distribution.

\section{Quiescent and star-forming dust clumps: clues to star formation processes and time scales}

The ATLASGAL survey provides an unbiased view of the embedded stages of massive star formation from the onset of collapse to the emergence of $\mathrm{H}$ II regions. Therefore it provides unprecedented statistics to study the properties of dust condensations in various stages: quiescent phase prior to the onset of collapse, as well as actively star-forming cores. Here we use these statistics to investigate the physical properties of the quiescent versus star-forming cores (Sect. 7.1), derive a global star formation rate for the Galaxy (Sect. 7.2), and provide a good estimate of time scales for these phases (Sect. 7.3).

\subsection{Properties of quiescent and star-forming clumps}

In Fig. 7 we show the flux density distribution of ATLASGAL sources with mid-infrared counterpart, i.e., embedded sources with ongoing star formation, corresponding to $33 \%$ of the ATLASGAL sources (i.e., both MSX and WISE associations). Their peak flux distribution is found to be very similar to that of all ATLASGAL sources, and we derive a slope of $\alpha=$ $-1.25 \pm 0.04$. This value suggests a shallower distribution of the peak fluxes compared to that of all ATLASGAL sources, although this is clearly due to the lower number of star-forming ATLASGAL sources at the lower peak flux-density range. We note that the distribution of the peak flux density exhibits this uniform scaling over more than two orders of magnitude for both star-forming and all ATLASGAL clumps. 
To further investigate the origin of this shallower distribution, in Fig. 18 we show the beam-averaged flux density versus the fraction of star-forming ATLASGAL sources compared to all sources. Clearly, the fraction of star-forming clumps increases with increasing peak flux density, suggesting that the vast majority of the brightest clumps are actively forming stars. This fraction seems to be a constant of $\sim 0.75$ within the errors for all sources above a beam-averaged flux density of $5 \mathrm{Jy}$, corresponding to $0.7-4.2 \times 10^{23} \mathrm{~cm}^{-2}$ column density for warm $\left(T_{\mathrm{d}}=30 \mathrm{~K}\right)$ and cold $\left(T_{\mathrm{d}}=10 \mathrm{~K}\right)$ gas. Adopting an average distance of $4.5 \mathrm{kpc}$ and a temperature of $18 \mathrm{~K}$, this threshold gives a similar value to the $650 M_{\odot}$ that we obtained from the extrapolation of the MDCs in Cygnus-X from Motte et al. (2007) to the ATLASGAL survey (see Sect. 3.5), suggesting that these sources could potentially sustain high-mass star formation. This increase in the fraction of star-forming sources may also indicate that there is a change in the star formation process in massive clumps, although this transition is likely smeared to a continuous trend by the distance effects in Fig. 18.

We point out that there are only a few massive ATLASGAL clumps above this threshold that do not seem to harbor midinfrared embedded sources. These massive, quiescent-looking sources are interesting because they may be either pristine massive clumps (i.e., the high-mass analogs of low-mass prestellar cores) at the onset of collapse processes. Alternatively, they can harbor low- to intermediate-mass embedded protostars (massive protostellar clumps, prior to the MYSO phase), which are too faint to be detected with WISE at $22 \mu \mathrm{m}$ at greater distances.

Investigating the appearance of these sources, we find a mean aspect ratio of 1.38 with a standard error of 0.005 for starforming ATLASGAL sources, while all sources have a mean of 1.49. A KS-test further rejects the null hypothesis that they are drawn from the same distribution with a significance level of $<0.0001$. This shows that sources hosting embedded protostars exhibit a rather spherical, less elongated morphology.

\subsection{The Galactic star formation rate}

Adopting the color estimates of Wood \& Churchwell (1989), similar to Motte et al. (2007) and Russeil et al. (2010), we extrapolate a flux density of $\sim 30 \mathrm{Jy}$ at $21 \mu \mathrm{m}$ at a distance of $1 \mathrm{kpc}$ for a B3 type star with $10^{3} L_{\odot}$ (see also Sect. 5.3), which corresponds to $\sim 75 \mathrm{mJy}$ on the far side of the Galaxy at $20 \mathrm{kpc}$. This is above the WISE sensitivity limit, therefore we are sensitive to all B3 and earlier type massive stars in the Galaxy. Based on the assumption that with WISE and MSX we cover all high-mass star-forming sites within $20 \mathrm{kpc}$, we derive a crude estimate here for the current rate of star formation for these deeply embedded objects.

The time scale for the protostellar evolutionary stage for high-mass stars is still being debated. If the accretion rate is constant and is on the order of the observed $\sim 10^{-4} M_{\odot} \mathrm{yr}^{-1}$ (Klaassen \& Wilson 2007), at least $10^{5}$ years or longer are needed to build up a $10 M_{\odot}$ star. On the other hand, there are a few studies suggesting that the time scale for high-mass protostars can be shorter than (e.g., Motte et al. 2007), or just as long (Duarte-Cabral et al. 2013) as for low-mass protostars, where a life-time on the order of $10^{5} \mathrm{yr}$ has been derived (Evans et al. 2009). Theoretical models predict a star formation time scale on the order of $10^{5} \mathrm{yr}$ (e.g., McKee \& Tan 2003; Offner \& McKee 2011). We therefore adopt the so far best observationally derived value of $\sim 3 \pm 1 \times 10^{5}$ yr by Duarte-Cabral et al. (2013) for the highly embedded stage and account for a multiplicity of $\sim 2$, which has been seen toward MDCs in Cygnus-X at high angular-resolution (Bontemps et al. 2010). We estimate

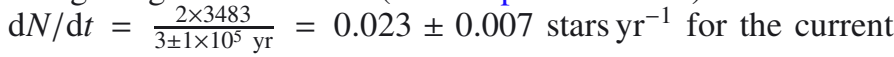
formation rate for high-mass stars. Assuming that they form on average 8-10 $M_{\odot}$ stars, we estimate a star formation rate of $0.21 \pm 0.07 M_{\odot} \mathrm{yr}^{-1}$ for massive stars.

Taking the IMF of Kroupa et al. (1993) and neglecting the brown-dwarf population (objects with $M<0.08 M_{\odot}$ ), $8.6 \%$ of the total mass is in OB-type stars with masses between 10-120 $M_{\odot}$. Based on this we can extrapolate the star formation rate derived above to a Galactic star formation rate of $\frac{0.21 \pm 0.07}{0.086}=2.44 \pm 0.81 M_{\odot} \mathrm{yr}^{-1}$. This estimate is based on the assumption that all the star-forming ATLASGAL sources form massive stars, which is certainly contaminated by intermediatemass nearby objects, and is at the same time also incomplete for the brightest sources saturated in both the MSX and WISE catalogs. Nevertheless, it provides an independent and comparable value for the global star formation rate in our Galaxy as derived by other methods. Using the YSO population revealed by Spitzer, Robitaille \& Whitney (2010) suggest a value between 0.68 to $1.45 M_{\odot} \mathrm{yr}^{-1}$, while Diehl et al. (2006) estimate $4 M_{\odot} \mathrm{yr}^{-1}$ based on radioactive ${ }^{26} \mathrm{Al}$ measurements. Comparing these different tracers in a homogenous way, Chomiuk \& Povich (2011) arrive to an estimate of $2 M_{\odot} \mathrm{yr}^{-1}$. Our estimate is consistent with these studies considering the large uncertainties in the exact number of clumps forming massive stars, the protostellar life-time estimates, and the poorly constrained factor of multiplicity.

\subsection{Formation time scales}

As discussed in Sect. 5.1, we expect our mid-infrared characterization to be more robust against chance alignments than that of Contreras et al. (2013). Other studies, such as the BGPS, base the mid-infrared diagnostics of their sources on the GLIMPSE and MSX point source catalogs. Dunham et al. (2011a) finds that $44 \%$ of the BOLOCAM sources have a mid-infrared counterpart; however, they consider a $50 \%$ chance alignment and arrive at a conservative estimate that $20 \%$ of the BGPS sources have an embedded mid-infrared source.

Our analysis (Sects. 5.1, 5.2) is based on requiring a detected source at $22 \mu \mathrm{m}$ by benefitting from a continuous sensitivity between WISE and MSX with a similar spatial resolution to the ATLASGAL survey, thereby restricting the number of chance alignments with field stars and nearby Class II/III YSOs that have no or only weak mid-infrared emission. Nevertheless, our sample can suffer from a minor contamination by AGB stars and other red sources that are not distinguishable from our sample based on their colors. That our estimate is more robust against chance alignments is also supported by the color-color plots, suggesting that indeed most of our matches are deeply embedded sources. As a consequence, our estimate of $\sim 33 \%$ is better constrained than previous studies.

When taking the $650 M_{\odot}$ limit for dense clumps to form massive stars, this translates to a $5 \mathrm{Jy}$ limit at a distance limit of $4.5 \mathrm{kpc}$. Assuming that all quiescent ATLASGAL sources above this limit are prone to collapse and will form massive stars, we estimate the time scale for the pre-collapse phase for the most massive clumps in the Galaxy. Adopting the estimate for a protostellar life time of $3 \pm 1 \times 10^{5} \mathrm{yr}$ (see also Sect. 7.2) and considering that only $\sim 25 \%$ of the sources are quiescent, this phase cannot last longer than $7.5 \pm 2.5 \times 10^{4} \mathrm{yr}$. This estimate is consistent with other studies (e.g., Motte et al. 2007, 2010; Russeil et al. 2010; Tackenberg et al. 2012) and is one order of magni- 
tude lower than that of low-mass cores (e.g., Enoch et al. 2007; Hatchell \& Fuller 2008), suggesting that massive cores (precursors of high-mass stars) form in a fast, dynamic process.

In the dynamic scenario, the formation time scale is dictated by the crossing time. Considering the above derived $7.5 \pm 2.5 \times$ $10^{4} \mathrm{yr}$ as the crossing time for the prestellar and Class 0 like embedded phases, and the average physical size of $0.4 \mathrm{pc}$, a velocity dispersion of $\sim 5.4 \pm 1.7 \mathrm{~km} \mathrm{~s}^{-1}$ is required. This is in good agreement with kinematic studies of high-mass starforming sites, where flows of dense gas have been observed at similar relative velocities (e.g., Schneider et al. 2010; Csengeri et al. 2011a,b; Nguyen-Lu'o'ng et al. 2013). This provides a coherent view of dynamical processes to be at the origin of massive star formation in our Galaxy.

\section{Summary}

We have produced a catalog of embedded sources in the ATLASGAL survey using a multiscale decomposition tool to remove extended emission and then used a Gaussian source-fitting algorithm (MRE-GCL, Motte et al. 2010). This method was optimized to identify the population of centrally condensed, compact structures. Here we summarize our main results:

1. We identified 10565 ATLASGAL sources in the main part of the survey, between $|\ell|<60^{\circ}$ and $|b|<1.5^{\circ}$. In the extension region, between $-80^{\circ}<\ell<-60^{\circ}$ and $-2^{\circ}<b<1^{\circ}$, with a higher average noise level we extracted 296 sources. Our catalog is complete to $>99 \%$ above $7 \sigma$.

2. We found good correlation in the distribution of peak flux density compared to the values from a different method by Contreras et al. (2013) that was optimized for larger sources corresponding to clumps and cloud structures. We derived a slope of $\alpha \sim 1.44 \pm 0.03$ for the distribution of the peak flux density, which was found to be consistent with other surveys, such as the BGPS.

3. We used the MSX and WISE point source catalogs to assess the star formation activity of the ATLASGAL sources and found that $33 \%$ of them harbor embedded mid-infrared sources. Color-color plots of the WISE-ATLASGAL matches demonstrated the characteristic reddened colors of these sources.

4. The Galactic distribution of ATLASGAL sources shows peaks toward the most prominent star-forming complexes in our Galaxy. We found that the star-forming sources exhibit similar distribution and that they similarly peak at the position of rich complexes. Considering all WISE sources with characteristic red color we found a good correlation between the ATLASGAL clumps and red objects, suggesting that star formation mainly takes place in large complexes.

5. We determined the Galactic scale height for the dust sources of $\sim 0.32^{\circ}$, which is less than previous estimates using midinfrared surveys; however, it is close to the value determined by surveys of young massive star-forming regions associated with $\mathrm{H}_{2} \mathrm{O}$ or methanol masers (Walsh et al. 2011; Urquhart et al. 2013a).

6. From the fraction of star-forming sources we estimate a Galactic star formation rate of $\sim 2.44 \pm 0.81 M_{\odot} \mathrm{yr}^{-1}$. Although this value is subject to large uncertainties due to both the unknown factor of multiplicity and the time scale for the protostellar evolutionary phase, we still find a good agreement with other estimates in the literature.

7. By comparing the fraction of star-forming versus quiescent ATLASGAL sources, we showed that the number of embedded sources exhibiting star formation activity increases with the beam-averaged flux density. This ratio is found to be a rather constant value of $75 \%$ for $>5 \mathrm{Jy}$ beamaveraged flux density, suggesting that the life time for the infrared-dark evolutionary phase is short. We estimate an upper limit for the infrared-dark phase of $7.5 \pm 2.5 \times 10^{4}$ years.

Acknowledgements. We thank the referee for comments that helped improve the manuscript. This work was partially funded by the ERC Advanced Investigator Grant GLOSTAR (247078) and was partially carried out within the Collaborative Research Council 956, subproject A6, funded by the Deutsche Forschungsgemeinschaft (DFG). L.B. acknowledges support from CONICYT project PFB-06. This paper is based on data acquired with the Atacama Pathfinder EXperiment (APEX). APEX is a collaboration between the Max Planck Institute for Radioastronomy, the European Southern Observatory, and the Onsala Space Observatory. This research made use of data products from the Midcourse Space Experiment. Processing of the data was funded by the Ballistic Missile Defense Organization with additional support from the NASA Office of Space Science. This research has also made use of the NASA/ IPAC Infrared Science Archive, which is operated by the Jet Propulsion Laboratory, California Institute of Technology, under contract with the National Aeronautics and Space Administration. This publication makes use of data products from the Wide-field Infrared Survey Explorer, which is a joint project of the University of California, Los Angeles, and the Jet Propulsion Laboratory/California Institute of Technology, funded by the National Aeronautics and Space Administration.

\section{Appendix A: Source parameters from Gaussclumps}

Gaussian decomposition procedures, such as Gaussclumps have been widely used in the literature, both on 3D cubes of molecular line observations, as well as 2D maps of dust emission. The reliability of the algorithm has been tested and discussed partly in Stutzki \& Güsten (1990) and more in detail in Kramer et al. (1998). We refer the reader to these papers for a detailed discussion of the algorithm.

However, since this is the largest survey where Gaussclumps has been applied, we performed several test to assess the reliability of the detections. In Sect. A.1 we discuss the radial profiles of the sources and the impact of the filtering on the measured sizes, while in Sect. A.2 we compare the extracted peak fluxes to the original values in the filtered and non-filtered maps.

\section{A.1. Sizes and radial flux density profiles}

Since with the filtering some background emission is removed from the maps, we investigated the impact of this on the measured sizes by performing source extraction on maps with different scales of background emission removed. The change in the azimuthally averaged flux density profile by using different scales, between $50-400^{\prime \prime}$, for the background is illustrated in Fig. A.1. By comparing the extracted sizes in these measurements, we found that the decrease in the measured sizes with respect to that of the original maps is negligible $(\sim 10-20 \%)$ for the 100 " filtering scale used here.

\section{A.2. Source parameters: the peak flux}

We have performed several tests to estimate the reliability of the source parameters, such as peak flux density and size extracted by Gaussclumps.

As a first step we compared the extracted peak fluxes with the fluxes in the filtered and the original maps. On average we find this difference to be less than $30 \%$ averaged on the individual tiles, however locally, in very complex regions this fraction can be higher. Figure A. 2 shows the ratio between the extracted and the measured peak flux density at the position of the source 

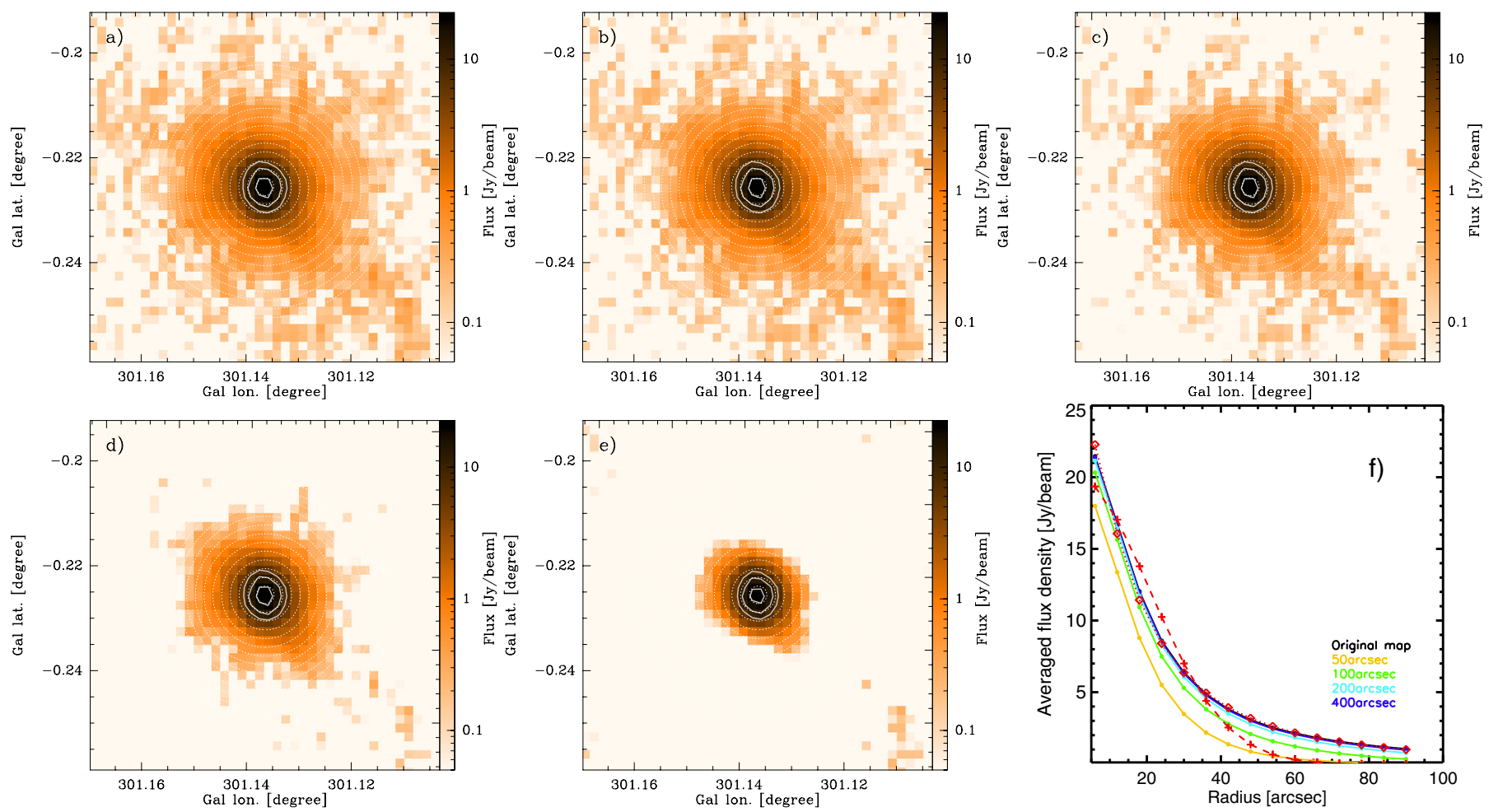

Fig. A.1. One of the brightest sources detected shown with different scales of filtering, similarly as in Fig. 2. The peak flux of the object in this case decreases by $20 \%$ from the original images to the most compact one, while the size decreases by only $10 \%$. Panel f) shows azimuthally averaged flux density profile with different scales of background emission removed. The green line corresponds to the filtering used for our catalog, which has $2 \times 50^{\prime \prime}$ as maximum scale. As a comparison a Gaussian profile is indicated in red dashed line with crosses and a dotted line with diamond symbols shows a power-law fit.

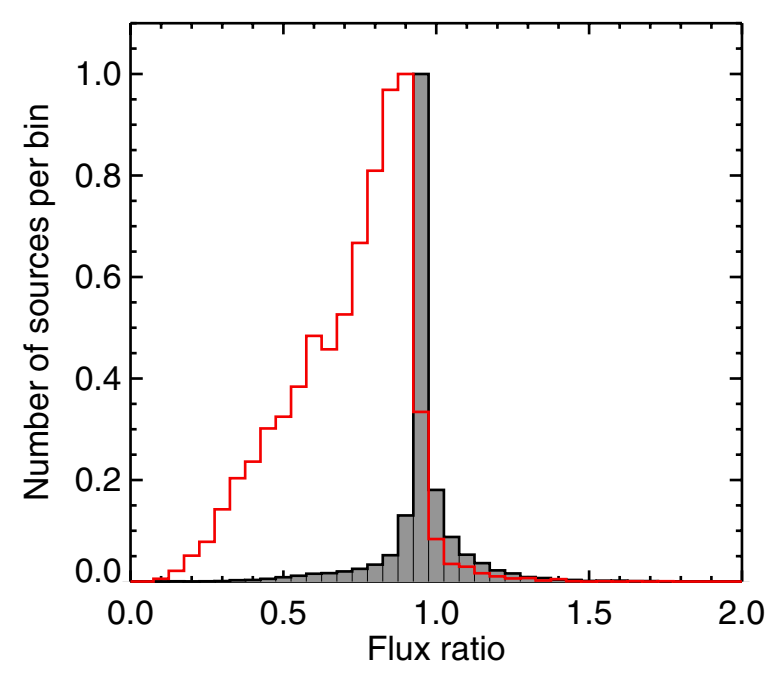

Fig. A.2. Normalized ratio of peak flux density calculated from the extracted (i.e. fitted) and the actual pixel values at the position of the source. Black histogram shows the ratio calculated using the filtered maps, while the red line shows the ratio calculated from the original maps with the total emission. (See Sect. A.2 for discussion.)

using the both the filtered and the original maps. The major fraction of the sources have peak flux density within $5 \%$ of the pixel values in the filtered maps confirming that the algorithm works good finding the peak position of the dust emission. On average the measured flux is $97.9 \%$ of the extracted value with a median of $98.2 \%$ (Fig. A.2). The difference comes from two factors: first, fluctuations in the local noise may lead to small shift in the determined positions, i.e. determining the peak biased towards a higher value pixel instead of the peak of the Gaussian profile. Hence the measured value in the actual pixel may differ from the fitted value. We find $\sim 500$ sources $(5.4 \%)$ of the total sources where this flux ratio differs with $>30 \%$ compared to unity. We inspected each of these sources and they are dominantly weak and large sources with no clear Gaussian distribution in which case the peak position is not well defined. On the other hand, since the algorithm decomposes sources in confused regions, we also find flux ratios below $100 \%$ which are blended sources. We find that a marginal fraction of the sources is actively deblended $(0.34 \%$ of the total number of sources), where within the beam the source is decomposed into two components. There are, however a larger fraction (24\%) of overlapping sources, which reflects the clumpy nature of the dust distribution in molecular clouds. Altogether we have a good census of the reliability of the extracted peak flux values of the algorithm.

The extracted peak flux densities compared to the values in the original emission maps show that the sources have on average $73.5 \%$ of the pixel value with a median of $78 \%$. This suggests that the filtering lowers on average the peak flux values by $20-30 \%$.

\section{Appendix B: Color-color plots of star-forming ATLASGAL clumps}

Figures B.1-B.4 show color-color plots and a color-magnitude diagrams of the ATLASGAL and WISE matched sources. Comparing the positions of embedded UC-H II regions, RMS and MMB sources in Fig. B.3 we see that especially the former two occupy a well determined region with bright $12 \mu \mathrm{m}$ flux and 


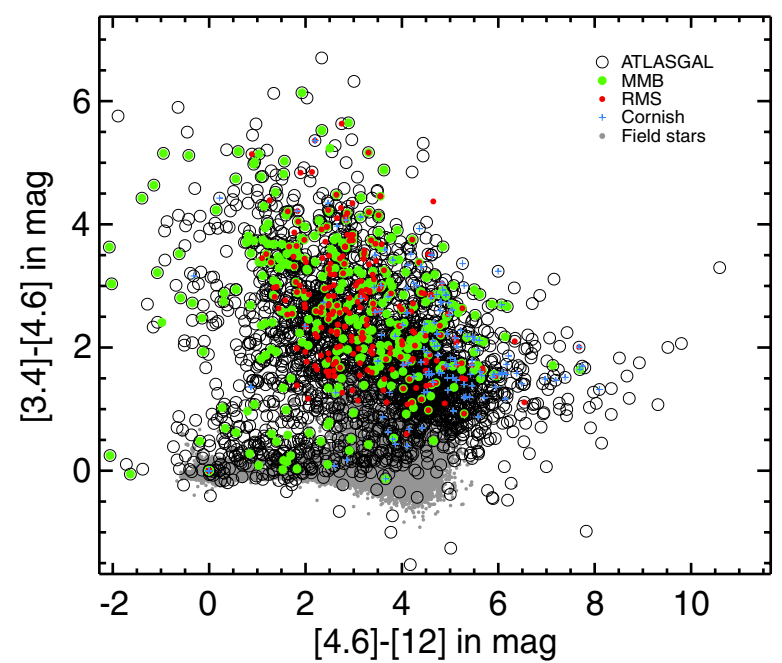

Fig. B.1. Color-color plots of ATLASGAL sources with a WISE source match (black dots). As a comparison, the colors of field stars from a test field are shown in gray dots.

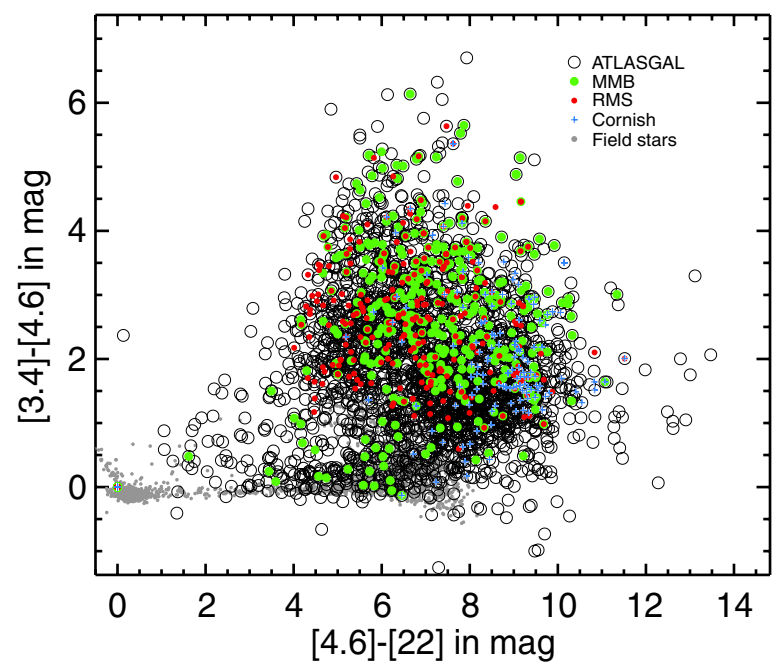

Fig. B.2. Color-color plots of ATLASGAL sources with a WISE source match (black dots). As a comparison, the colors of field stars from a test field are shown in gray dots.

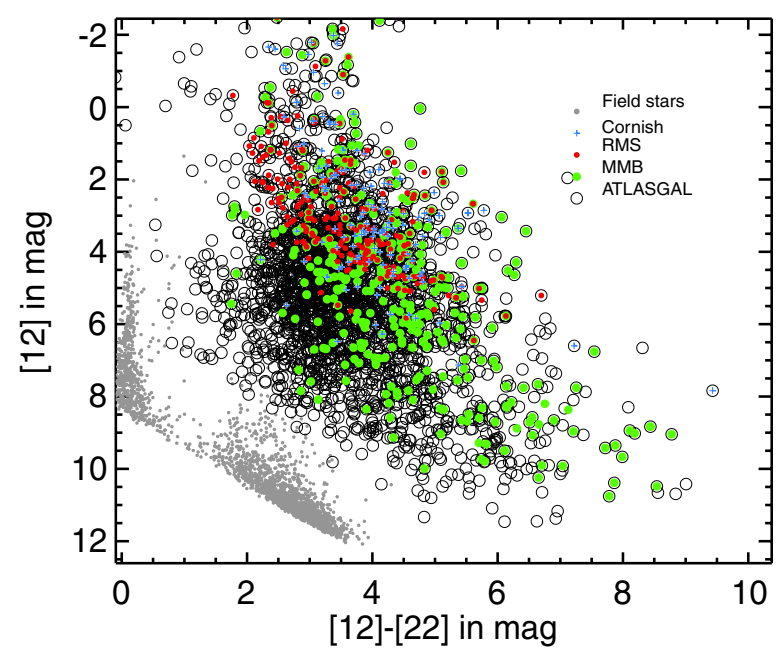

Fig. B.3. Color-magnitude plots of ATLASGAL sources with a WISE source match (black dots). As a comparison, the colors of field stars from a test field are shown in gray dots.

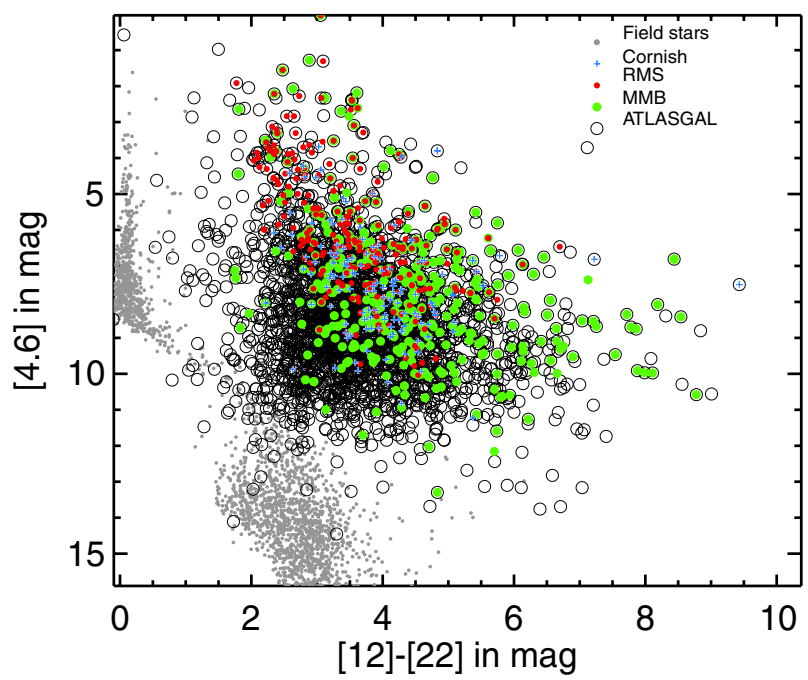

Fig. B.4. Color-magnitude plots of ATLASGAL sources with a WISE source match (black dots). As a comparison, the colors of field stars from a test field are shown in gray dots.

colors between $1.8<[12]-[22]<7$. These sources correspond to evolved stages, $\mathrm{H}$ II regions and MYSOs. These two samples seem to have no distinguishable color properties.

\section{References}

Aguirre, J. E., Ginsburg, A. G., Dunham, M. K., et al. 2011, ApJS, 192, 4 André, P., Ward-Thompson, D., \& Barsony, M. 2000, in Protostars and Planets IV (Tucson: University of Arizona Press), 59

André, P., Men'shchikov, A., Bontemps, S., et al. 2010, A\&A, 518, L102 Becker, R. H., White, R. L., Helfand, D. J., \& Zoonematkermani, S. 1994, ApJS, 91, 347

Benjamin, R. A., Churchwell, E., Babler, B. L., et al. 2003, PASP, 115, 953 Bergin, E. A., \& Tafalla, M. 2007, ARA\&A, 45, 339

Bernard, J.-P., Paradis, D., Marshall, D. J., et al. 2010, A\&A, 518, L88

Bertin, E., \& Arnouts, S. 1996, A\&AS, 117, 393

Beuther, H., Schilke, P., Menten, K. M., et al. 2002, ApJ, 566, 945

Beuther, H., Tackenberg, J., Linz, H., et al. 2012, ApJ, 747, 43

Bontemps, S., Motte, F., Csengeri, T., \& Schneider, N. 2010, A\&A, 524, A18

Bronfman, L., Cohen, R. S., Alvarez, H., May, J., \& Thaddeus, P. 1988, ApJ, 324,248

Bronfman, L., Nyman, L.-A., \& May, J. 1996, A\&AS, 115, 81

Bronfman, L., Casassus, S., May, J., \& Nyman, L.-Å. 2000, A\&A, 358, 521

Butler, M. J., \& Tan, J. C. 2009, ApJ, 696, 484

Carey, S. J., Clark, F. O., Egan, M. P., et al. 1998, ApJ, 508, 721

Carey, S. J., Noriega-Crespo, A., Mizuno, D. R., et al. 2009, PASP, 121, 76

Chomiuk, L., \& Povich, M. S. 2011, AJ, 142, 197

Cohen, R. S., \& Thaddeus, P. 1977, ApJ, 217, L155

Contreras, Y., Schuller, F., Urquhart, J. S., et al. 2013, A\&A, 549, A45

Csengeri, T., Bontemps, S., Schneider, N., Motte, F., \& Dib, S. 2011a, A\&A, 527, A 135

Csengeri, T., Bontemps, S., Schneider, N., et al. 2011b, ApJ, 740, L5

Cutri, R. M., Wright, E. L., Conrow, T., et al. 2012, Explanatory Supplement to the WISE All-Sky Data Release Products, Tech. Rep.

Deharveng, L., Schuller, F., Anderson, L. D., et al. 2010, A\&A, 523, A6

Di Francesco, J. 2008, in AAS Meeting Abstracts \#212, BAAS, 40, 271

Diehl, R., Halloin, H., Kretschmer, K., et al. 2006, Nature, 439, 45

Duarte-Cabral, A., Bontemps, S., Motte, F., et al. 2013, A\&A, 558, A125

Dunham, M. K., Robitaille, T. P., Evans, II, N. J., et al. 2011a, ApJ, 731, 90

Dunham, M. K., Rosolowsky, E., Evans, II, N. J., Cyganowski, C., \& Urquhart, J. S. 2011b, ApJ, 741, 110

Egan, M. P., Shipman, R. F., Price, S. D., et al. 1998, ApJ, 494, L199

Egan, M. P., Price, S. D., Cohen, M., et al. 1999, Techn. Rep. AFRL-VS-TR1999-1522, (Springfield: NTIS)

Egan, M. P., Price, S. D., Kraemer, K. E., et al. 2003, VizieR on-line Data Catalog: 5/114, AFRL-VS-TR-2003-1589, Air Force Research Laboratory

Enoch, M. L., Glenn, J., Evans, II, N. J., et al. 2007, ApJ, 666, 982

Evans, II, N. J. 1999, ARA\&A, 37, 311

Evans, II, N. J., Dunham, M. M., Jørgensen, J. K., et al. 2009, ApJS, 181, 321 
Foster, J. B., Jackson, J. M., Barnes, P. J., et al. 2011, ApJS, 197, 25 Gaume, R. A., Johnston, K. J., \& Wilson, T. L. 1993, ApJ, 417, 645 Green, J. A., Caswell, J. L., Fuller, G. A., et al. 2009, MNRAS, 392, 783 Güsten, R., Nyman, L. A., Schilke, P., et al. 2006, A\&A, 454, L13

Gwinn, C. R., Moran, J. M., \& Reid, M. J. 1992, ApJ, 393, 149

Hatchell, J., \& Fuller, G. A. 2008, A\&A, 482, 855

Hoare, M. G., Lumsden, S. L., Oudmaijer, R. D., et al. 2005, in Massive Star Birth: A Crossroads of Astrophysics, eds. R. Cesaroni, M. Felli, E. Churchwell, \& M. Walmsley, IAU Symp., 227, 370

Hoare, M. G., Purcell, C. R., Churchwell, E. B., et al. 2012, PASP, 124, 939 Hughes, V. A., \& MacLeod, G. C. 1989, AJ, 97, 786

Immer, K., Menten, K. M., Schuller, F., \& Lis, D. C. 2012, A\&A, 548, A120 Jackson, J. M., Rathborne, J. M., Foster, J. B., et al. 2013, PASA, 30, e057 Johnstone, D., Wilson, C. D., Moriarty-Schieven, G., et al. 2000, ApJ, 545, 327

Johnstone, D., Fich, M., Mitchell, G. F., \& Moriarty-Schieven, G. 2001, ApJ, 559,307

Kainulainen, J., Beuther, H., Banerjee, R., Federrath, C., \& Henning, T. 2011, A\&A, 530, A64

Kauffmann, J., \& Pillai, T. 2010, ApJ, 723, L7

Kauffmann, J., Bertoldi, F., Bourke, T. L., Evans, II, N. J., \& Lee, C. W. 2008, A\&A, 487, 993

Kennicutt, Jr., R. C. 1998, ARA\&A, 36, 189

Klaassen, P. D., \& Wilson, C. D. 2007, ApJ, 663, 1092

Kramer, C., Stutzki, J., Rohrig, R., \& Corneliussen, U. 1998, A\&A, 329, 249

Kroupa, P., Tout, C. A., \& Gilmore, G. 1993, MNRAS, 262, 545

Kruijssen, J. M. D., Longmore, S. N., Elmegreen, B. G., et al. 2014, MNRAS, 440,3370

Lada, C. J., \& Lada, E. A. 2003, ARA\&A, 41, 57

Ladjal, D., Justtanont, K., Groenewegen, M. A. T., et al. 2010, A\&A, 513, A53

Longmore, S. N., Bally, J., Testi, L., et al. 2013, MNRAS, 429, 987

López, C., Bronfman, L., Nyman, L.-Å., May, J., \& Garay, G. 2011, A\&A, 534, A131

Lumsden, S. L., Hoare, M. G., Oudmaijer, R. D., \& Richards, D. 2002, MNRAS, 336,621

Lumsden, S. L., Hoare, M. G., Urquhart, J. S., et al. 2013, ApJS, 208, 11

McKee, C. F., \& Tan, J. C. 2003, ApJ, 585, 850

Men'shchikov, A., André, P., Didelon, P., et al. 2012, A\&A, 542, A81

Minier, V., André, P., Bergman, P., et al. 2009, A\&A, 501, L1

Molinari, S., Brand, J., Cesaroni, R., \& Palla, F. 1996, A\&A, 308, 573

Molinari, S., Swinyard, B., Bally, J., et al. 2010a, A\&A, 518, L100

Molinari, S., Swinyard, B., Bally, J., et al. 2010b, PASP, 122, 314

Molinari, S., Schisano, E., Faustini, F., et al. 2011, A\&A, 530, A133

Mookerjea, B., Kramer, C., Nielbock, M., \& Nyman, L.-Å. 2004, A\&A, 426, 119

Motte, F., \& Hennebelle, P. 2009, in EAS Publ. Ser. 34, eds. L. Pagani, \& M. Gerin, 195

Motte, F., André, P., \& Neri, R. 1998, A\&A, 336, 150

Motte, F., André, P., Ward-Thompson, D., \& Bontemps, S. 2001, A\&A, 372, L41

Motte, F., Schilke, P., \& Lis, D. C. 2003, ApJ, 582, 277

Motte, F., Bontemps, S., Schilke, P., et al. 2007, A\&A, 476, 1243
Motte, F., Zavagno, A., Bontemps, S., et al. 2010, A\&A, 518, L77 Nguyen Luong, Q., Motte, F., Schuller, F., et al. 2011, A\&A, 529, A41 Nguyen-Lu'o'ng, Q., Motte, F., Carlhoff, P., et al. 2013, ApJ, 775, 88 Offner, S. S. R., \& McKee, C. F. 2011, ApJ, 736, 53

Ossenkopf, V., \& Henning, T. 1994, A\&A, 291, 943

Perault, M., Omont, A., Simon, G., et al. 1996, A\&A, 315, L165

Peretto, N., \& Fuller, G. A. 2009, A\&A, 505, 405

Peretto, N., \& Fuller, G. A. 2010, ApJ, 723, 555

Peretto, N., André, P., \& Belloche, A. 2006, A\&A, 445, 979

Peretto, N., Fuller, G. A., Plume, R., et al. 2010, A\&A, 518, L98

Pillai, T., Wyrowski, F., Carey, S. J., \& Menten, K. M. 2006, A\&A, 450, 569

Price, S. D., Egan, M. P., Carey, S. J., Mizuno, D. R., \& Kuchar, T. A. 2001, AJ, 121,2819

Purcell, C. R., Hoare, M. G., Cotton, W. D., et al. 2013, ApJS, 205, 1

Ragan, S., Henning, T., Krause, O., et al. 2012, A\&A, 547, A49

Rathborne, J. M., Jackson, J. M., \& Simon, R. 2006, ApJ, 641, 389

Reid, M. J., Menten, K. M., Zheng, X. W., et al. 2009, ApJ, 700, 137

Robitaille, T. P., \& Whitney, B. A. 2010, ApJ, 710, L11

Robitaille, T. P., Meade, M. R., Babler, B. L., et al. 2008, AJ, 136, 2413

Rosolowsky, E., Dunham, M. K., Ginsburg, A., et al. 2010, ApJS, 188, 123

Russeil, D., Zavagno, A., Motte, F., et al. 2010, A\&A, 515, A55

Rygl, K. L. J., Wyrowski, F., Schuller, F., \& Menten, K. M. 2010, A\&A, 515, A42

Sato, M., Reid, M. J., Brunthaler, A., \& Menten, K. M. 2010, ApJ, 720, 1055

Schneider, N., Csengeri, T., Bontemps, S., et al. 2010, A\&A, 520, A49

Schuller, F. 2012, in SPIE Conf. Ser., 8452

Schuller, F., Omont, A., Glass, I. S., et al. 2006, A\&A, 453, 535

Schuller, F., Menten, K. M., Contreras, Y., et al. 2009, A\&A, 504, 415

Simon, R., Rathborne, J. M., Shah, R. Y., Jackson, J. M., \& Chambers, E. T. 2006, ApJ, 653, 1325

Simpson, J. P., Cotera, A. S., Burton, M. G., et al. 2012, MNRAS, 419, 211

Siringo, G., Kreysa, E., Kovács, A., et al. 2009, A\&A, 497, 945

Sridharan, T. K., Beuther, H., Schilke, P., Menten, K. M., \& Wyrowski, F. 2002, ApJ, 566, 931

Starck, J.-L., \& Murtagh, F. 2006, Astronomical Image and Data Analysis (Springer-Verlag)

Stutzki, J., \& Güsten, R. 1990, ApJ, 356, 513

Tackenberg, J., Beuther, H., Henning, T., et al. 2012, A\&A, 540, A113

Urquhart, J. S., Busfield, A. L., Hoare, M. G., et al. 2008, A\&A, 487, 253

Urquhart, J. S., Moore, T. J. T., Hoare, M. G., et al. 2011, MNRAS, 410, 1237

Urquhart, J. S., Moore, T. J. T., Schuller, F., et al. 2013a, MNRAS, 431, 1752

Urquhart, J. S., Thompson, M. A., Moore, T. J. T., et al. 2013b, MNRAS, 435 400

Vasyunina, T., Linz, H., Henning, T., et al. 2009, A\&A, 499, 149

Walsh, A. J., Breen, S. L., Britton, T., et al. 2011, MNRAS, 416, 1764

Wienen, M., Wyrowski, F., Schuller, F., et al. 2012, A\&A, 544, A146

Wilcock, L. A., Ward-Thompson, D., Kirk, J. M., et al. 2012, MNRAS, 422, 1071

Williams, J. P., de Geus, E. J., \& Blitz, L. 1994, ApJ, 428, 693

Wood, D. O. S., \& Churchwell, E. 1989, ApJ, 340, 265

Wright, E. L., Eisenhardt, P. R. M., Mainzer, A. K., et al. 2010, AJ, 140, 1868 